\title{
ALIANZAS Y ESTRATEGIAS DE LEGITIMACIÓN DE LA NOBLEZA INDÍGENA EN EL NORTE DE YUCATÁN DURANTE EL POSCLÁSICO: UN ENFOQUE COMPARATIVO Y MULTIDISCIPLINARIO
}

\author{
María FloRes HernÁndeZ \\ Manuel Eduardo Pérez Rivas \\ Dirección de Salvamento Arqueológico, \\ Instituto Nacional de Antropología e Historia
}

Resumen: Durante la época prehispánica, los grupos indígenas que ostentaron el poder político desarrollaron diversas estrategias para legitimar y mantener sus privilegios respecto al resto de la población. En el norte de Yucatán, estas prácticas datan de los Ahauob del Clásico. Sin embargo, también hubo innovaciones e integración de elementos de otras áreas, como el centro de México. Datos actuales sugieren que durante el Posclásico aparecieron nuevas formas de gobierno, donde la jerarquía política y organización territorial recayeron más que nunca en el ámbito de relaciones humanas que no perduran en el contexto arqueológico. En este artículo se presentan resultados de una investigación enfocada en las formas de interacción de la nobleza indígena. Mediante unificación de datos y técnicas de la historia, arqueología y geografía (SIG), se definieron patrones característicos que guardan relación con el discurso de origen del poder y la estructura política y territorial de las unidades políticas o cuchcabalob del Posclásico.

Palabras clave: Ah Kin Ch’el, Posclásico, Yucatán, linajes, sig.

AвSTRACT: During pre-Hispanic times, indigenous groups who held political power developed various strategies to legitimize and maintain their privileges with the rest of the population. In the North of Yucatan, some of these practices date back to the Ahauob of the Classic period. However, there were also innovations and integration of elements from other areas such as Central Mexico. Current data suggest that during the Postclassic new government forms emerged, where political and territorial organization hierarchy fell more than ever in the field of human relations which not always left archaeological traces. We present results of a research focused on understanding the interaction of the indigenous nobility or almehenob. Through the integration of data and techniques from history, archeology and geography (GIS), we identified patterns that correlate with the narratives of legitimacy and source of power, and also the political and territorial structure of the polities or cuchcabalob of the Postclassic.

Kerwords: Ah Kin Ch'el, Postclassic, Yucatan, lineages, sIG.

RECEPCIÓN: 30 de agosto del 2010.

ACEPTACIÓN: 23 de febrero del 2011. 



\title{
ALIANZAS Y ESTRATEGIAS DE LEGITIMACIÓN DE LA NOBLEZA INDÍGENA EN EL NORTE DE YUCATÁN DURANTE EL POSCLÁSICO: UN ENFOQUE COMPARATIVO Y MULTIDISCIPLINARIO
}

\author{
María Flores HernándeZ \\ Manuel Eduardo Pérez Rivas \\ Dirección de Salvamento Arqueológico, \\ Instituto Nacional de Antropología e Historia
}

En el ámbito de la investigación mayista siempre ha existido interés en lograr definir las características y extensión de las unidades políticas prehispánicas; en especial, los mecanismos de interacción entre los individuos que constituían el estamento dominante de la sociedad. Los estudios de patrón de asentamiento, así como los estudios y lecturas epigráficas, han constituido una importante fuente de hipótesis para tratar de entender los sistemas de gobierno de las entidades políticas mayas.

Aunque aún hay dudas sobre las características y extensión de las unidades políticas del periodo Clásico, la figura de los gobernantes supremos o ahauob y sus historias dinásticas han sido objeto de exhaustivos estudios. ${ }^{1}$ La manera como los gobernantes han sido representados a través del tiempo ha sido el punto de partida para proponer cambios en la manera como era ejercido el poder político. De acuerdo a las evidencias actuales, durante el Clásico Tardío / Terminal se pasó de una relativa verticalidad de las decisiones de gobierno a una estructura administrativa más compleja que dependía de alianzas y acuerdos con otros personajes de la nobleza indígena. La aparición cada vez más frecuente de señores subordinados y cargos políticos secundarios en las inscripciones de este periodo son un reflejo de este fenómeno (Martin y Grube, 1995: 42). Esta situación se acentúa durante el Posclásico, donde, a la par del surgimiento de nuevos cargos centrales, como el halach uinic, ${ }^{2}$ se dan cambios sustanciales en las

\footnotetext{
"Nota del editor. Los gobernantes mayas del Clásico recibían, entre otros, el título de K'uhul Ajaw. Se ha respetado la ortografía de los autores en las palabras mayas.

${ }^{1}$ Véanse por ejemplo Schele y Freidel, 1990; Demarest, 1992; Marcus, 1976, 1993; Grube y Martin, 1998; Martin y Grube, 2000.

${ }^{2}$ El título de halach uinic o jalach winik no se ha registrado en las inscripciones mayas del periodo Clásico (véase Kettunen y Helmke, 2010). En este trabajo se ha respetado en lo posible la ortografía
} 
formas de gobierno, que han sido relacionados con migraciones e influencias de otras áreas de Mesoamérica, en especial del centro de México.

En nuestra investigación, partimos del supuesto de que, durante esta última fase de la historia prehispánica de Yucatán, más que un fenómeno de decadencia o desintegración cultural, las nuevas formas de gobierno, así como los elementos de jerarquización y organización territorial, recayeron más que nunca en el ámbito de las relaciones humanas y mecanismos de circulación asimétrica de bienes y trabajo humano. ${ }^{3}$ Frecuentemente su materialización no siempre perduró en el contexto arqueológico. Probablemente, para reforzar estos vínculos a través de la redistribución de objetos como presentes o regalos, se incrementó la participación directa de los gobernantes del Posclásico en las redes de intercambio.

Los resultados que se presentan provienen de una investigación enfocada en el centro norte de Yucatán, específicamente en lo que Ralph Roys definió como la "provincia" o cuchcabal de Ah Kin Ch'el y sus vecinos, donde, a través de la conjugación de datos y técnicas procedentes de la historia, arqueología y geografía (SIG), se establecieron pautas y patrones característicos de asociación entre los linajes indígenas principales, los cuales guardan relación con el discurso de origen del poder y la estructura política y territorial particular de las diferentes unidades políticas, o cuchcabalob, del Posclásico. ${ }^{4}$

En el desarrollo de esta investigación se tomaron como fuente de hipótesis las ideas sobre la organización política y territorial de los mayas propuesta por Quezada (1993) y Okoshi $(1992,1994)$ contrastándolas con las evidencias arqueológicas e históricas sobre Ah Kin Ch'el. Los resultados mostraron algunas diferencias con los planteamientos de estos autores. En particular, nuestra interpretación

colonial de los términos mayas. Por ello, palabras glotalizadas como el apellido Ch'el se escriben así por la dificultad topográfica de representar la "h" herida (Chel).

${ }^{3}$ La designación misma del periodo Posclásico siempre ha estado asociada a la connotación de declive con respecto al Clásico (Sharer, 1994: 384-386). Las diferencias contrastantes en manifestaciones culturales como la calidad de la arquitectura y la cerámica han sido elementos base para sustentar procesos de desintegración política y "decadencia cultural” vinculados con la migraciones de distintos grupos como los "Itzáes" (Roys, 1962: 41-46). Autores como Sabloff y Freidel (1975) han interpretado las características de la arquitectura Posclásica como resultado de una orientación mercantil y "pragmática" de las élites del Posclásico. Por su parte, Susan Kepecs (1999), utilizando la teoría de "sistemas mundiales", sugiere que este fenómeno está relacionado con procesos como la descentralización del poder, con énfasis en el comercio y el incremento de la competencia entre las entidades políticas del Posclásico Tardío y Terminal.

${ }^{4}$ En este trabajo utilizaremos "provincia" para referirnos a una división territorial sujeta a algún tipo de autoridad administrativa o de gobierno. Sin embargo, para evitar posibles confusiones con el concepto colonial hispano de "provincia", evitaremos en lo posible utilizarlo para referirnos a las entidades políticas mayas del Postclásico, prefiriendo entonces el uso de área o región en un sentido geográfico amplio (véase Okoshi, 1992: 272). Jurisdicción sería una noción equivalente, refiriéndose al término de un lugar circunscrito al mando de alguna autoridad. En el ámbito político territorial utilizaremos el término cuchcabal, retomando las dos connotaciones o campos semánticos establecidas por Okoshi, 1997: 1) los gobernantes y oficiales que el señor supremo tiene a su cargo en un espacio territorial y 2) un espacio territorial gobernado por un poder que reside en un lugar determinado (Okoshi, 1997: 2). 
con respecto al desarrollo y conformación de la jurisdicción política de los Ch'el difiere en varios aspectos de la planteada por Sergio Quezada con base en su análisis de documentación histórica.

Un elemento que mostró el análisis fue que los mecanismos para el establecimiento de nuevos linajes, y su legitimación, presentan algunas similitudes y posibles influencias con casos específicos del centro de México, las que se han revisado e incluido en un primer nivel de comparación.

\section{Los esquemas de alianza política en el norte de Yucatán}

A través de la arqueología, la reconstrucción del panorama político para una etapa temporal determinada es difícil y resulta siempre fragmentaria, puesto que hay detalles o aspectos de las relaciones humanas, territoriales y políticas que no se materializan en el contexto arqueológico. Por ejemplo, sólo con datos arqueológicos es difícil encontrar indicios materiales que nos sugieran la presencia o dominio de determinado linaje o grupo familiar en un sitio específico. Mucho más complicado es obtener evidencias directas de posibles alianzas o vínculos políticos entre dos o más asentamientos (plano 1). Esto es más palpable en el norte de Yucatán donde, en el mejor de los casos, los análisis de datos y materiales sólo pueden proporcionar una aproximación. Aquí toma sentido la complementariedad del empleo sistemático y metódico de los registros escritos cualesquiera que sea su formato (documentación colonial, códices, inscripciones, etc.).

En este trabajo ha resultado provechoso aplicar algunas técnicas del ámbito de la arqueología al análisis de documentos del periodo Colonial Temprano. En general, estas técnicas se fundamentan básicamente en principios de asociación o relación temporal y espacial, así como en comparaciones y elaboración de tipologías de entidades y artefactos. A través de la adecuación de este método se examinó detalladamente la ubicación geográfica de los linajes nobles en Ah Kin Ch'el mediante el diseño e integración de una base de datos con registros de los gobernadores y principales indígenas conocidos desde época prehispánica hasta 1607 (tabla 1). ${ }^{5}$ Entre los primeros resultados obtenidos, se corroboró que los

\footnotetext{
${ }^{5}$ Los puntos de partida para la integración de esta base de datos fueron la obra de Roys (1957) y la compilación del Apéndice 2 de la obra de Quezada (1993). No obstante, se revisó y amplió la información consultando directamente microfilms de algunos de los documentos de archivo y otras fuentes publicadas. Entre ellos podemos mencionar los siguientes: Relaciones Histórico Geográficas de la Gobernación de Yucatán (1983); Carta en donde los caciques piden a SM., religiosos de la orden de San Francisco (1567, Archivo General de Indias [AGI], Audiencia de México, referencia ES.41091. AGI/1.16403.13.369//MEXICO,367); Tasaciones de los pueblos de la provincia de Yucatán hechas por la audiencia de Santiago de Guatemala en el mes de febrero de 1549 (AGI, Papeles de Simancas, Caj. 6, Leg. 1) (AGI, Audiencia de Guatemala, Leg. 128, ff. 307-401v); Residencia tomada al doctor Diego de Quijada, del tiempo que fue gobernador de la provincia de Yucatán, Cozumel y Tabasco, por Luis de Céspedes y Oviedo, juez nombrado para este efecto y gobernador de dichas provincia ( AGI Justicia $245 \mathrm{f} 1001 \mathrm{v}-1562 \mathrm{v}$. Microfilm consultado en el archivo del Centro de Estudios Mayas (Сем), Universidad Nacional Autónoma de México (UNAM). Código de referencia del AGI: ES.41091.AGI/23.8.26/JUSTICIA,245).
} 


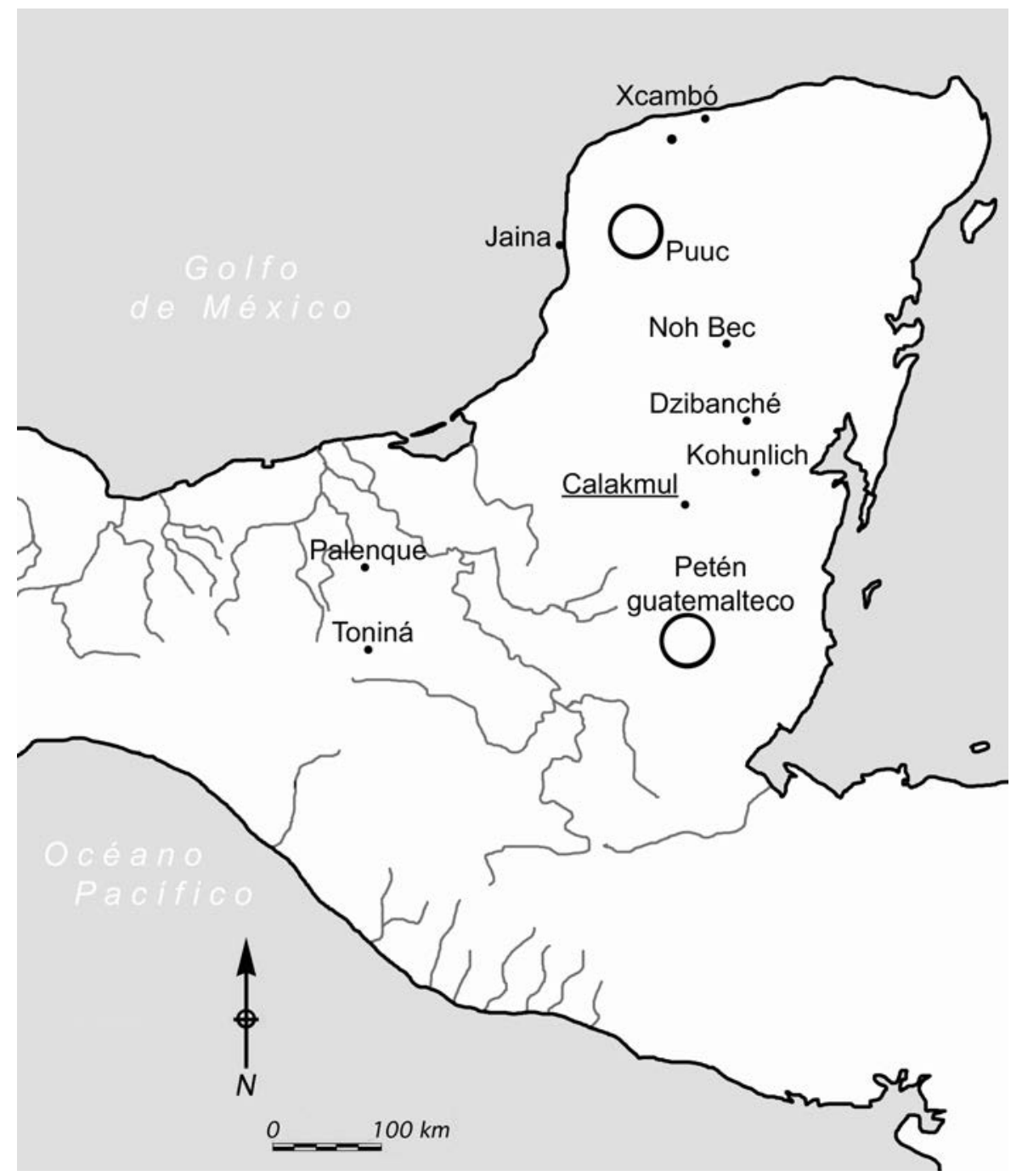

Plano 1. Área de estudio y sitios arqueológicos

efectos del régimen colonial no se reflejaron fuertemente durante los primeros dos tercios del siglo xvI. ${ }^{6}$ El seguimiento de los batabob y los gobernadores indígenas mostró continuidad en los nombres de los linajes principales que persistieron en el gobierno de sus pueblos hasta 1565-1567 (tabla 2).

${ }^{6}$ El estudio de Quezada (1993) muestra cómo se transformó gradualmente el sistema de organización indígena a través del periodo Colonial. Una de las estrategias básicas para eliminar la influencia de la nobleza indígena dentro de los pueblos fue la paulatina substitución de los batabob y los miembros de su linaje por gobernadores indígenas y miembros de cabildo que no estaban relacionados con la estructura de poder prehispánica. 


\begin{tabular}{|c|c|c|c|}
\hline \multicolumn{2}{|c|}{ RESIDENCIA DE QUIJADA 1365} & \multicolumn{2}{|c|}{ DATOS COMBINADOS DE DIVERSAS FUENTES 1565-1567 } \\
\hline Patronímico & Frecuencia & Patronímico & Frecuencia \\
\hline Pech & 9 & Pech & 23 \\
\hline Cupul & 4 & luit & 5 \\
\hline luit & 4 & Cupul & 4 \\
\hline Cocom & 3 & Cocom & 3 \\
\hline Tun & 3 & Ch'el & 3 \\
\hline Cauich & 2 & Tun & 3 \\
\hline Dzul & 2 & Cauich & 2 \\
\hline Ek & 2 & Ek & 2 \\
\hline Motul & 2 & Motul & 2 \\
\hline Pot & 2 & Pot & 2 \\
\hline U can & 2 & U can & 2 \\
\hline fké & 1 & "é & 1 \\
\hline FJatun & 1 & Batún & 1 \\
\hline Cahun & 1 & Cahum & 1 \\
\hline Camal & 1 & Camal & 1 \\
\hline Canché & 1 & Can & 1 \\
\hline Canul & 1 & Canché & 1 \\
\hline Chan & 1 & Canul & 1 \\
\hline Che & 1 & Che & 1 \\
\hline Ch'el & 1 & Chin & 1 \\
\hline Chin & 1 & Chuil P & 1 \\
\hline Chuil & 1 & Dzib & 1 \\
\hline Dzib & 1 & Dzul & 1 \\
\hline Fernández & 1 & Euán & 1 \\
\hline Hau & 1 & Fernández & 1 \\
\hline Huchim & 1 & Hau & 1 \\
\hline bí & 1 & Huchim & 1 \\
\hline Kantun & 1 & bí & 1 \\
\hline$M^{\wedge}$ in $o M^{\wedge}$ un & 1 & Mazin o Mazun & 1 \\
\hline Nagual & 1 & Nagual & 1 \\
\hline Ruíz Pech & 1 & Oxté & 1 \\
\hline Tzab & 1 & Tzab & 1 \\
\hline Uc & 1 & Uicab & 1 \\
\hline Uicab & 1 & Uto & 1 \\
\hline Uto & 1 & & \\
\hline Cuenta general & 59 & Cuenta general & 74 \\
\hline
\end{tabular}

Tabla 1. Frecuencias de individuos que ostentaban cargo de gobernador indígena en el área de estudio 
En el área de estudio ocurrieron cambios en 13 de 78 pueblos (tabla 3, plano 2). ${ }^{7}$ Esto sugiere que, a pesar de las transformaciones ocurridas, los gobernadores indígenas y miembros de los cabildos correspondían mayoritariamente a los almehenob o noblezas indígenas locales del Posclásico. Por tanto, suponemos que el arreglo, distribución y frecuencia de estos personajes, sobrevivientes de la nobleza indígena y miembros de un sector de la sociedad que aún controlaba el poder político, son útiles para entender con más detalle la geografía política prehispánica del área estudiada, en especial de posibles alianzas o pugnas por el control de ciertos pueblos. ${ }^{8}$

Los resultados se obtuvieron a partir de un análisis de correlaciones espaciales de los patronímicos de los principales indígenas de 1565 a $1567 .{ }^{9}$ La muestra fue de 429 individuos procedentes de 59 pueblos (tabla 4). Se registraron 125 apellidos o patronímicos con una frecuencia de repetición de 1 a 31. Se elaboraron planos de concentraciones y estimaciones de distribución geográfica por medio de algoritmos de interpolación, ${ }^{10}$ a partir de los siguientes supuestos básicos:

- Los patronímicos más extensos geográficamente y con una mayor frecuencia pueden considerarse como locales o con una antigüedad de poblamiento mucho mayor que los menos frecuentes. Los Pech y los Cupul serían casos típicos de esta distribución.

- Patronímicos relativamente escasos y con una concentración espacial muy específica pudieran considerarse como de posible origen foráneo o con una menor antigüiedad temporal en la región. En este patrón encajan la distribución de linajes como los Ch'el, los luit y los Cocom. Otra posibilidad es considerar a los patronímicos escasos como residual de una población desplazada en el pasado; sin embargo, en este caso el patrón lógico espe-

\footnotetext{
${ }^{7}$ Buctzotz, Citilcum, Dzilam, Hoctún, Temax, Tepakán, Teya, Uitzil, Usil, Tinum, Tibolón, Yaxcabá y Xocchel, ver plano 2.

${ }^{8}$ Esto es particularmente importante con respecto al linaje Ch'el, ya que su frecuencia es escasa en números absolutos y en cantidad de pueblos gobernados directamente, lo que sugiere que debieron recurrir a una serie de alianzas y mecanismos de coerción para mantener el control del cuchcabal en época prehispánica. Por ejemplo, en la Residencia de Quijada sólo aparece registrado Francisco Ch'el como gobernador de Dzidzantún, aunque por otras fuentes sabemos que para este momento había personajes de apellido Ch'el en el gobierno de los pueblos de Yobaín y Cansahcab

${ }^{9}$ El material principal se obtuvo del documento Residencia tomada al doctor Diego de Quijada, del tiempo que fue gobernador de la provincia de Yucatán, Cozumel 1 y Tabasco, por Luis de Céspedes y Oviedo, juez nombrado para este efecto y gobernador de dichas provincias. AGI Justicia 245 f1001v-1562v. Microfilm consultado en el archivo del CEM UNAM. Código de referencia del AGI: ES.41091.AGI/23.8.26/JUSTICIA,245

${ }^{10}$ Este tipo de algoritmos, como el kriging, empleados frecuentemente en software de sistemas de información geográfica, topografía y manejo de imágenes, permite la obtención o estimación de datos nuevos con base en la distribución, frecuencia y ordenamiento de los datos de origen. En el caso que nos ocupa, el resultado son mapas predictivos o de estimación que muestran tendencias con base en la frecuencia y localización geográfica de los personajes que comparten el mismo apellido en un área geográfica.
} 


\begin{tabular}{|c|c|c|c|c|}
\hline \multirow{2}{*}{\begin{tabular}{|l|}
$I D$ \\
18 \\
\end{tabular}} & \multirow{2}{*}{\begin{tabular}{|l|} 
Pueblo \\
Tekantó \\
\end{tabular}} & \multirow{2}{*}{\begin{tabular}{|l} 
Patronímicos registrados \\
de 1565-1567
\end{tabular}} & \multicolumn{2}{|c|}{$\begin{array}{l}\begin{array}{l}\text { Frecuencias de patronímicos de } \\
\text { gobernadores }\end{array} \\
\text { a }\end{array}$} \\
\hline & & & Ch'el & 3 \\
\hline 24 & TixLial & 3 atún & Pech & 3 \\
\hline \multirow[t]{2}{*}{6} & Citilcum & Cahum & Cupul & 2 \\
\hline & Aclunich & Camal & Chan & 2 \\
\hline 16 & Tecal & Canché & IUbtul & 2 \\
\hline 7 & Dzilam (San Francisco) & Can-Pech & Tun & 2 \\
\hline 20 & Tepakan (San Juan Evangelista) & Cauich & Ucan & 2 \\
\hline 4 & Chalante (San Miguel) & Cupul & Aké & 1 \\
\hline 40 & Yalsihón & Cupul & Balam & 1 \\
\hline 8 & zamal & Che & Batún & 1 \\
\hline 3 & Cansahcab San Francisco) & Ch'el & Cahum & 1 \\
\hline 1 & Dzidzantún & Ch'el & Canal & 1 \\
\hline 29 & Yobaín & Ch'el & Can & 1 \\
\hline 14 & Sitilpech & Chin & Canché & 1 \\
\hline 23 & Tixculum (San Pedro) & Dzib & Cauich & 1 \\
\hline 9 & Kantunil & Dzul & Che & 1 \\
\hline 19 & Temax & Ek-Chan-Ek & Chin & 1 \\
\hline 13 & Sinanché (San Buenaventura) & Euán & Dzfc & 1 \\
\hline 28 & Xanabá & Huchim & Dzul & 1 \\
\hline 5 & Chaltunpuhuy & Motul & Ek & 1 \\
\hline 15 & $\begin{array}{l}\text { Sudzal (La Asunción de } \\
\text { Nuestra Señora) }\end{array}$ & Motul & Euán & 1 \\
\hline 10 & Kimbilá (Santa Clara) & Pech & Huchim & 1 \\
\hline 27 & Uitzil & Pech-Chan & Macin & 1 \\
\hline 63 & Sahcaba (en Tecoh) & Pot-Dzul & Pol & 1 \\
\hline 22 & Tixcochoh & Tun & Pot & 1 \\
\hline 21 & Teva (San Bernabé Apóstol) & Tun-P d & Uicab & 1 \\
\hline 12 & Pomolché (San Idelfonso) & Ucan & Uitz & 1 \\
\hline 2 & Buctzotz (La Pura Concepción) & Ucan-Balam & & \\
\hline 25 & Tocbadz & Uicab & & \\
\hline 11 & Pixilá (San Buenaventura) & Uitz & & \\
\hline 17 & Tecoh & Macin? & & \\
\hline \multicolumn{5}{|c|}{ Pueblos registrados } \\
\hline \multicolumn{5}{|c|}{ Patronímicos de gobernantes indígenas } \\
\hline
\end{tabular}

Tabla 2. Patronímicos de gobernantes en Ah Km Ch’el para el periodo 1565-1967 


\begin{tabular}{|c|c|c|c|}
\hline$I D$ & Pueblo & Cttcit cabal & $\begin{array}{l}\text { Sustitución o alternada en el gobiemo del } \\
\text { pueblo }\end{array}$ \\
\hline 2 & Buctiotí & Ah Kin Ch'el & Ucan-Balain-Uitz \\
\hline 6 & Citilcum & Ah Kin Ch'el & Can-Cahum \\
\hline 4 & Chalante & Ah Kin Ch'el & Cupul-Tepal \\
\hline 7 & Diilam & Ah Kin Ch'el & Ch'el-Can-Pech \\
\hline 9 & Kantunil & Ah Kin Ch'el & Diul-Tu-Chin \\
\hline 12 & Pomolché & Ah Kin Ch'el & Ucan-Balam \\
\hline 63 & Saneaba & Ah Kin Ch'el & Pot-Diul \\
\hline 18 & Tekantó & Ah Kin Ch'el & Pot-Ake-Pot \\
\hline 19 & Temax & Ah Kin Ch'el & Ek-Chan-EK \\
\hline 20 & Tepakán & Ah Kin Ch'el & Couoh-Cauich-Chin-M o \\
\hline 21 & Teya & Ah Kin Ch'el & Tun-Pol \\
\hline 24 & Tixtual & Ah Kin Ch'el & Batun-Diul \\
\hline 27 & Uitiil & Ah Kin Ch'el & Pecli-Clian-Pecli-Chan \\
\hline 28 & Xanabá & Ah Kin Ch'el & Huchim-Ku \\
\hline 29 & Yobaín & Ah Kin Ch'el & Cli'el-Clian-Pech \\
\hline 49 & Baca & Cehpech & Pech-Che \\
\hline 80 & Mococha & Cehpech & Pech-Ciau \\
\hline 39 & Tinum & Cupul & Cupul-Kantun \\
\hline 42 & Mocaba & Mocaba & luit-Cen \\
\hline 43 & Hoctún & Mocaba & luit-Uc \\
\hline 45 & Huhí & Mocaba & luit-Puch \\
\hline 62 & Saneaba & Mocaba & Pot-luit \\
\hline 66 & Sanahcat & Mocaba & Tiab-Mo \\
\hline 44 & Tiscabanchel & Mocaba & luit-Ku \\
\hline 36 & Xocchel & Mocaba & Che-luit \\
\hline 46 & Tibolón & Sotuta & Ix-Chuil \\
\hline 65 & Usil & Sotuta & Tun-Yah-Cauich-Pech \\
\hline 61 & Yaxcabá & Sotuta & Pecli-Ku-Pech-Ku-H.iu \\
\hline
\end{tabular}

Tabla 3. Alternadas y sustituciones de linajes gobernantes en el área de estudio durante el siglo xVI 


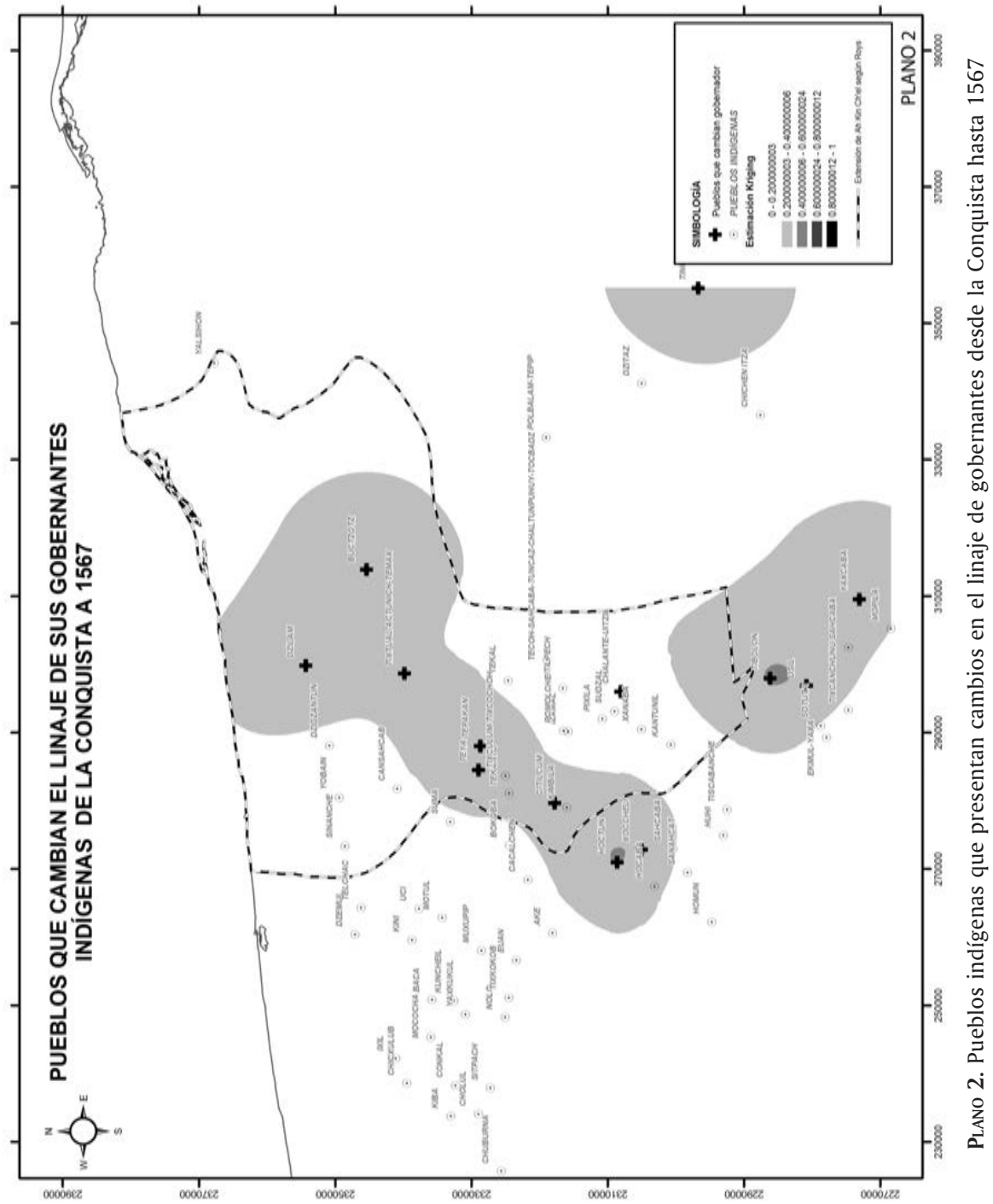




\begin{tabular}{|c|c|c|}
\hline Patronímico & Frecuencia & $\begin{array}{r}\text { Normalización por } \\
\text { Unidades de Desviación } \\
\text { Estándar }(U D E)\end{array}$ \\
\hline Peen & 31 & $6.3155956 * 16$ \\
\hline May & 19 & 3.5664977 \\
\hline Chan & 17 & $3.1083145-12$ \\
\hline Canul & 13 & 2.191348227 \\
\hline Can & 12 & $1.9628566-18$ \\
\hline Cocom & 12 & $1.9628566-18$ \\
\hline Dzul & 12 & 1.9628566-18 \\
\hline Canché & 11 & 1.733765069 \\
\hline Couoh & 11 & 1733765069 \\
\hline Pot & 11 & 1.733765059 \\
\hline $\mathrm{B}: \mathrm{I}, \mathrm{i} \sim *$ & 10 & 1.50467349 \\
\hline Dzib & 10 & 1.50467349 \\
\hline Cauich & 9 & 1.275581911 \\
\hline Tun & 9 & 1.275581911 \\
\hline Ek & 8 & 1.046493332 \\
\hline Uc & 8 & 1.046493332 \\
\hline $\mathrm{U}>\mathrm{cab}$ & 8 & $1.016-193332$ \\
\hline Baz & 7 & 0.817393753 \\
\hline luít & 7 & 0.817393753 \\
\hline Akó & 6 & 0.588337175 \\
\hline Chin & 6 & 0.588307175 \\
\hline Chuc & 6 & 0.588307175 \\
\hline $\mathrm{Be}$ & 5 & D_¿59ZZli595 \\
\hline Cupjl & 5 & 0.359215595 \\
\hline Che & 5 & 0.359215595 \\
\hline Chi & 5 & 0.359215595 \\
\hline Kantun & 5 & 0.359215595 \\
\hline Noh & 5 & 0.359215596 \\
\hline C:-vij-i & A & 0.130124017 \\
\hline Cantal & 4 & 0.130124017 \\
\hline Cen & 4 & 0.130124017 \\
\hline Euan & 4 & 0.130124017 \\
\hline Haj & 4 & 0.130124017 \\
\hline Ku & 4 & 0.130124017 \\
\hline Mocul & 4 & 0.130124017 \\
\hline Ucan & 4 & 0.130124017 \\
\hline Us & 4 & 0.130124017 \\
\hline Yan o Yam & 4 & 0.130124017 \\
\hline $\mathrm{Zj} \mathrm{j}$ & 4 & 0.130124017 \\
\hline Batun & 3 & -3.098957562 \\
\hline Cuc & 2. & -3.098957562 \\
\hline$C^{\prime}-\mathrm{u}$ & 3 & -0.098957552 \\
\hline
\end{tabular}

\begin{tabular}{|l|l|}
\hline Patronímico & Frecuencia \\
& \\
\hline Huchim & 3 \\
\hline Mex & 3 \\
\hline Cante & 2 \\
\hline Coba & 2 \\
\hline Cumu & 2 \\
\hline Cuy & 2 \\
\hline Cuz & 2 \\
\hline C hablé & 2 \\
\hline Choben & 2 \\
\hline Hah & 2 \\
\hline Kauíl & 1 \\
\hline Mena & 1 \\
\hline Motul & 1 \\
\hline Na & 1 \\
\hline Oy & 1 \\
\hline Prsté & 1 \\
\hline Puc & 1 \\
\hline Tuyú & 1 \\
\hline Ak & 1 \\
\hline Aki & 1 \\
\hline Bacab & 1 \\
\hline Cab & 2 \\
\hline Cah & 2 \\
\hline Cal & 1 \\
\hline Camaz? & 1 \\
\hline Cap & 1 \\
\hline Qima & 1 \\
\hline Cob & 1 \\
\hline Coc & 1 \\
\hline Coh & 1 \\
\hline Coy & 1 \\
\hline Coyi & 1 \\
\hline Guau & 1 \\
\hline Cul & 1 \\
\hline Chel & 1 \\
\hline Chen & 1 \\
\hline Cn:na & 1 \\
\hline Chinab & 1 \\
\hline Chücm & 1 \\
\hline Dzi & 2 \\
\hline
\end{tabular}

Tabla 4. Patronímicos de principales y miembros de los cabildos indígenas en el área de estudio, según datos de la residencia del doctor Diego Quijada 


\begin{tabular}{|c|}
\hline $\begin{array}{r}\text { Normalización por } \\
\text { Unidades de Desviación } \\
\text { Estándar }(U D E)\end{array}$ \\
\hline-0.098967562 \\
\hline-0.098957532 \\
\hline-0.328359141 \\
\hline-0.328359141 \\
\hline-0.328359141 \\
\hline$-0.32 \& 359141$ \\
\hline$-3.32 \& 359141$ \\
\hline-3.328359141 \\
\hline-0.328059141 \\
\hline-0.328059141 \\
\hline$-0.32 \& 359141$ \\
\hline-0.328059141 \\
\hline-0.328359141 \\
\hline-0.328059141 \\
\hline-0.328359141 \\
\hline-0.328359141 \\
\hline-0.328359141 \\
\hline-0.328359141 \\
\hline-0.55715072 \\
\hline-0.55715072 \\
\hline-0.55715072 \\
\hline-0.55715072 \\
\hline-0.55715072 \\
\hline-0.55715072 \\
\hline-0.55715072 \\
\hline-0.55715072 \\
\hline-3.55715072 \\
\hline-3.55715072 \\
\hline-3.55715072 \\
\hline-0.55715072 \\
\hline-0.55715072 \\
\hline-0.55715072 \\
\hline-3.55715072 \\
\hline-3.55715072 \\
\hline-0.55715072 \\
\hline-0.55715072 \\
\hline-0.55715072 \\
\hline-0.55715072 \\
\hline-3.55715072 \\
\hline-0.55715072 \\
\hline-0.55715072 \\
\hline-0.55715072 \\
\hline
\end{tabular}

\begin{tabular}{|c|c|c|}
\hline Patronímico & Frecuencia & $\begin{array}{r}\text { Normalización por } \\
\text { Unidades de Desviación } \\
\text { Estándar (UDE) }\end{array}$ \\
\hline Emal & 1 & -3.55715372 \\
\hline Fernández & 1 & -0.55715372 \\
\hline Ho & 1 & ^3.55715372 \\
\hline Horra & 1 & -3.55715372 \\
\hline be & 1 & ^ 3.55715372 \\
\hline Ke & 1 & -0.55715372 \\
\hline $\mathrm{Ki}$ & 1 & -0.55715372 \\
\hline Maj in & 1 & -0.55715372 \\
\hline Moc & 1 & -0.55715372 \\
\hline $\mathrm{Mu}$ & 1 & -0.55715072 \\
\hline Naqua & 1 & -0.55715372 \\
\hline Noc & 1 & -0.55715372 \\
\hline Pacab & 1 & -0.55715372 \\
\hline Pan? & 1 & -0.55715372 \\
\hline Pat & 1 & -0.55715372 \\
\hline Pet & 1 & ^3.55715372 \\
\hline Po & 1 & ^3.55715372 \\
\hline Puch & 1 & -0.55715372 \\
\hline Que & 1 & -0.55715372 \\
\hline Ouiab & 1 & -0.55715372 \\
\hline Ouicab? & 1 & -0.55715072 \\
\hline Ouime & 1 & -0.55715072 \\
\hline Ruiz Pech & 1 & -0.55715372 \\
\hline Tan muy & 1 & -0.55715372 \\
\hline Tat & 1 & -0.55715372 \\
\hline $\mathrm{Te}$ & 1 & -0.55715372 \\
\hline Ten $>$ coh & 1 & -3.55715372 \\
\hline Tep & 1 & -3.55715372 \\
\hline To & 1 & ^3.55715372 \\
\hline Tut & 1 & -0.55715372 \\
\hline Tuz & 1 & -0.55715372 \\
\hline Tzab & 1 & -0.55715372 \\
\hline Tzec & 1 & -0.55715372 \\
\hline Urtz & 1 & -0.55715372 \\
\hline Xcan & 1 & -3.55715372 \\
\hline Xin & 1 & -0.55715072 \\
\hline $\mathrm{Xu}$ & 1 & -0.55715372 \\
\hline xd: & 1 & -0.55715372 \\
\hline Yab & 1 & -3.55715372 \\
\hline Ya! & 1 & -0.55715372 \\
\hline Yet & 1 & -0.55715372 \\
\hline
\end{tabular}

\begin{tabular}{|l|l|l|}
\hline Suma & 429 & \\
\cline { 1 - 2 } $\begin{array}{l}\text { No. de } \\
\text { Patronímicos }\end{array}$ & 125 & \\
\cline { 1 - 2 } Media & 3.432 & \\
\cline { 1 - 2 } Mediana & 1 & \multirow{2}{*}{6.315596646} \\
\hline Desv. Est. & 4.3650666 & -0.55715072 \\
\hline Valor Max & 31 & \\
\hline Valor Min & 1 & \\
\hline
\end{tabular}


rado sería de pequeñas concentraciones dispersas a lo largo de una gran extensión geográfica.

- Los principales indígenas que comparten un mismo apellido no necesariamente pertenecían a un mismo linaje o casa señorial ${ }^{11}$ ni estaban obligados a depender de la misma unidad política. Sin embargo, las posibilidades de interrelación por afinidad homónima pueden ser más frecuentes que entre individuos con patronímicos diferentes. Es más probable la existencia de algún vínculo de parentesco cuando indígenas de un mismo patronímico presentan proximidad espacial en poblaciones contiguas. ${ }^{12}$ Aunque la existencia de un posible parentesco no asegura de facto un vínculo político, la afinidad por parentesco o simplemente homónima proporcionaba un potencial mayor para establecer relaciones políticas. Landa nos menciona que los yucatecos

[...] tienen mucha cuenta con saber el origen de sus linajes, especialmente si vienen de alguna casa de Mayapán; y eso procuran saberlo de los sacerdotes, que es una de sus ciencias, y jáctanse mucho de lo varones que en sus linajes ha habido señalados. ${ }^{13}$

Este mismo cronista denota la existencia de un vínculo de solidaridad entre los individuos de un mismo apellido. Así,

[...] a sus hijos e hijas los llamaban del nombre del padre y de la madre; el del padre como propio, y de la madre como apelativo; desta manera, el hijo de Chel [Ch'el] y Chan llamaban Na-chan-Chel [Na Chan Ch'el], que quiere decir hijos de fulanos y esta es la causa que dicen los indios son los de un nombre deudos y se tratan por tales; por eso cuando vienen en parte no conocida y necesitado, luego acude al nombre, y si hay quien, luego con toda caridad le reciben y tratan... ${ }^{14}$

Este vínculo de afinidad aunque no lleva a asumir lazos directos de parentesco o políticos entre los individuos que comparten un mismo apellido, sí sustenta un principio simple de solidaridad entre homónimos que puede ser aprovechado en el ámbito de relaciones políticas más complejas. ${ }^{15}$

El análisis estadístico mostró 18 patronímicos con una frecuencia superior a ocho individuos, que podrían asumirse como de origen local: Pech, May, Chan,

${ }^{11}$ En el sentido enunciado por Luis Reyes (1988) para el centro de México y donde se aprecian semejanzas muy interesantes con la élites mayas del Posclásico.

${ }^{12}$ Tal como puede observarse con los miembros del linaje Pech en las Crónicas de Yaxkukul (1926) y Chacxulubchen (1936).

${ }^{13}$ Landa, 1938. Cap. XXIV: 42-43.

14 Ibid.

${ }^{15}$ Cabe aclarar que no se está adoptando el concepto de organización parentil a la manera que lo hace Quezada con el tzucub. La propuesta de Sergio Quezada sólo considera la distribución geográfica de patronímicos de gobernadores de los pueblos de indios. 
Canul, Can, Cocom, Dzul, Canché, Couoh, Pot, Balam, Dzib, Cauich, Tun, Ek, Uc y Uicab (tabla 4). La etimología maya de los patronímicos apoya la suposición anterior, con excepción de Cocom, lo cual se abordará adelante. Algunos de estos apellidos mayoritarios como May, Dzul, Canché, Pot y Ek se distribuyen de manera generalizada en toda el área de estudio, demostrando que se trata probablemente de linajes locales cuya filiación política depende más de su ubicación geográfica que de vínculos políticos a un cuchcabal específico. Los demás patronímicos mayoritarios muestran una distribución más localizada o regionalizada, lo que fundamenta la existencia de posibles vínculos políticos y de parentesco regionales; algunos de ellos, enfocados en un solo cuchcabal.

Dentro del universo de 125 patronímicos de principales o almehenob, solamente un número restringido ocupaba posiciones de gobernador indígena (35, $29.60 \%$ del total), indicativo de que el grupo humano que podía convertirse en batab era aún más selecto y que no todos los nobles o almehenob podían ser batabob de sus pueblos. Destacan 17 patronímicos poco frecuentes ${ }^{16}$ que corresponden únicamente a puestos de gobernadores, lo que confirmaría su preponderancia política a pesar de su escasez: Ch'el, Motul, Ix, Maçin o Mazun, Nagual, Tzab, Uitz y Fernández.

\section{Asociaciones espaciales por cuchcabal y linaje gobernante}

En los planos generados, se registraron distribuciones significativas de patronímicos de indígenas principales, cuyos miembros mantenían el control de al menos un cuchcabal a principios del siglo xvi: Ch'el (Ah Kin Ch'el), Cocom (Sotuta), Cupul (Cupul), Iuit (Hocabá) y Pech (Cehpech). Dichos linajes se concentran sistemáticamente en el área donde uno de sus miembros gobernaba como halach uinic; o bien, varios de ellos mantenían el control sobre un conjunto de pueblos, revelando correspondencia general entre ubicación geográfica y su poder político. También se definieron asociaciones exclusivas y predominantes con otros linajes secundarios, dando indicios de posibles alianzas (plano 3). Destacan las siguientes:

Patronímicos asociados a Cehpech: Pech.

Patronímicos asociados a Cupul: Cupul, Uc.

Patronímicos asociados espacialmente con los Ch'el: Ch'el, Camal, Motul y

Mex (Asociación exclusiva); Dzib, Cauich, Cahun, Che, Mocul (asociación predominante).

Patronímicos asociados a Hocabá: Iuit, Noh.

Patronímicos asociados a Sotuta: Cocom, Be, Chí, Chuc, Kantún, Ku, Us.

Patronímicos asociados con Sotuta y Ah Kin Ch'el: Euan, Uicab, Balam.

${ }^{16}$ Menos de 4 individuos principales registrados en total (ver tabla 1): Cahun, Camal, Hau, Ucan, Batun, Chuil, Huchim, Motul, Ch'el, Ix, Maçin, Nagual, Ruiz Pech, Tzab y Uitz. 


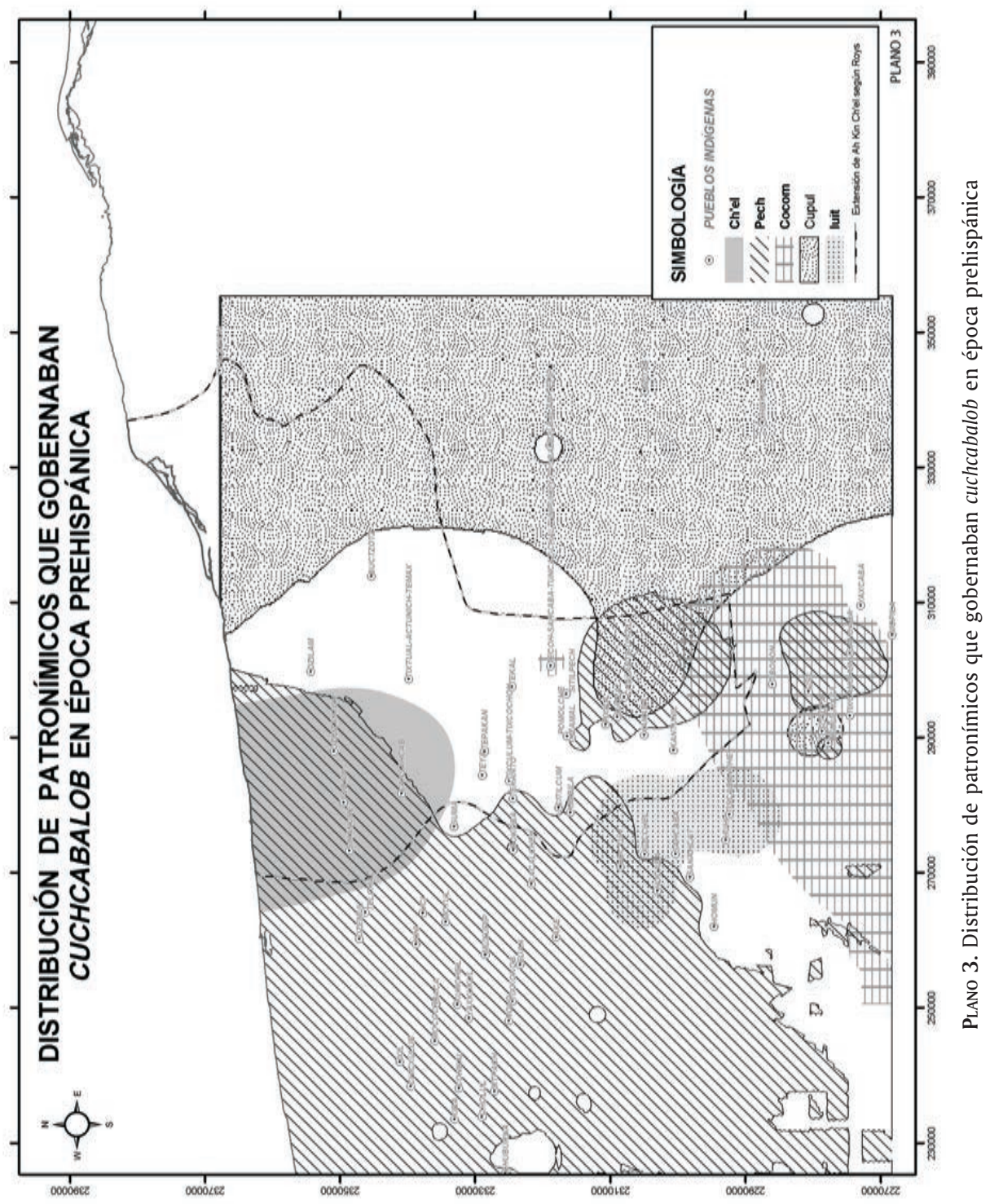


Patronímicos asociados a Sotuta-Hocabá: Canul, Can, Couoh, Zulu.

Patronímicos asociados a Hocabá-Sotuta-Ah Kin Ch'el: Chan, Baz y Tun.

Patronímicos con amplia distribución espacial: Aké, Ek, Canché, Dzul, May, Pot.

\section{Alianzas y su relación con la legitimación del poder.}

La integración de los análisis de planos junto con otras informaciones arqueológicas e históricas permitió esbozar un primer panorama de las alianzas y rivalidades del área estudiada. Se determinó que hay un esquema estrechamente vinculado al discurso de origen y legitimidad del poder para cada linaje en particular. Sólo abordaremos dos aspectos relacionados con este tema. El primero se trata del posible origen foráneo o local de los linajes gobernantes del área estudiada. En los textos coloniales hay distinciones entre los "señores naturales" y aquellos que gobiernan por provenir de un lugar diferente. ${ }^{17}$ Aunque es posible que algunos linajes del norte de Yucatán tuvieran o no un origen verdaderamente foráneo, lo importante es que dicha procedencia, ficticia o no, se convierte en un fuerte elemento de distinción para validar su acceso al poder político. ${ }^{18}$ La distribución de linajes observada, así como la etimología de sus apellidos, guarda coherencias significativas en algunos casos.

El segundo aspecto se relaciona con la serie de etapas o "pruebas" por las que pasan los fundadores de estos linajes "foráneos" para ser reconocidos como gobernantes legítimos. Independientemente de los simbolismos rituales que guardan estos discursos de origen y legitimación, hay acciones enmascaradas que dejan entrever una estrategia para establecer y consolidar el dominio político de una región. Las referencias a obtener el poder "más por maña que por fuerza", "ofrecerse en servicio", ser "criado" o "yerno" de algún gobernante legítimo, también son mencionadas, y con más detalle, en algunos casos del Posclásico del centro de México. Esta estrategia funcionó, aparentemente con éxito, para algunos linajes de Yucatán, que en sus inicios probablemente no tenían un derecho legítimo de gobernar por sucesión, como pudiera ser el caso de los Xiu, los luit, los Ch'el e incluso los fundadores del gobierno de Mayapán. ${ }^{19}$

\footnotetext{
${ }^{17}$ Entreverándose con aspectos míticos y simbólicos, las crónicas indígenas reiteran una serie de migraciones que dan origen a los pueblos dominantes. La "pequeña bajada del oriente" (cenial) y la "gran bajada del poniente" (nohenial) son un ejemplo de esto (Lizana, 1995: 61; Molina Solís, 1943, V.I: 3). También es frecuente la atribución de un origen extranjero para las élites gobernantes, como el poniente de Zuyuá de los Itzáes y el Tulapan Chiconahthan-Nonoual de los Xiu (Códice Pérez, 1949: 264-265). En documentos como las Relaciones Histórico Geográficas de la Gobernación de Yucatán (1983) se consigna que grupos como los Canul decían venir "del poniente de Zuyuá"; los Pech, del "oriente”; y los Cupul, también del oriente o de "México".

${ }^{18}$ Véase a Helms (1998: xi, citado en Restall, 2006: 269-270).

${ }^{19}$ En uno de sus escritos Tsubasa Okoshi (1996) ha demostrado cómo en los documentos coloniales indígenas se presentan ejemplos de discursos de legitimación acordes con la visión prehispánica.
} 


\section{Las alianzas de los Pech}

Los Pech son el linaje más abundante y predominante de toda la región de estudio. Las frecuencias más altas se concentran en el cuchcabal de Cehpech, extendiéndose hacia el sureste, donde los cuchcabalob vecinos. La importancia política regional se observó en el alto número de personajes con cargo de gobernador, casi todos pertenecientes a pueblos de Cehpech. La distribución es una tendencia prácticamente continua, y sólo se interrumpe en una franja formada por los poblados de Tisculum, Tixcochoh, Citilcum, Xocchel y Kantunil, la mayoría de ellos pertenecientes a Ah Kin Ch'el. A excepción de Dzidzantún, Izamal, Dzudzal y Chalamté, los Pech como principales no están presentes en los demás pueblos de Ah Kin Ch'el para 1565 (plano 4).

¿Esta exclusión entre los Pech y los pueblos de Ah Kin Ch'el podría reflejar alguna rivalidad o competencia por el control de regiones específicas? Las evidencias apuntan hacia esta interpretación. Resulta significativo que el vacío en la tendencia de expansión del patronímico Pech sea ocupado por apelativos vinculados casi exclusivamente con Ah Kin Ch'el, y prácticamente ausentes de los pueblos de Cehpech. Los apellidos que cumplen estas características serían Mocul, Euan, Chí, Can, Noh, Chan, Dzib y Tun. Complementariamente, durante el último tercio del siglo xVI y principios del xvII, cuando se acentúa la descomposición de los sistemas prehispánicos de organización social y política, especialmente el de Ah Kin Ch'el, se observa una substitución de miembros del linaje Ch'el por gente de apellido Pech en el gobierno de algunos pueblos principales como Dzidzantún (1567) y Yobaín (1607) (Quezada, 1993: 178).

Ningún otro patronímico se asocia de manera directa con los Pech, reflejando el amplio dominio que mantenían sobre su cuchcabal, controlando los vínculos y asociaciones políticas con otros linajes al interior y exterior de Cehpech. Esta exclusividad es evidente en la gubernatura de sus pueblos, donde solamente 2

\footnotetext{
En éstos destacan los simbolismos que asocian al gobernante con el Sol, los rumbos cardinales, o como el eje o puente entre los diferentes planos del cosmos y la continuidad del linaje. En esta estructura simbólica suelen aparecer pruebas que los personajes deben pasar antes de ser reconocidos como gobernantes. La distinción de un origen extranjero, el manejo de conocimientos y virtudes sacerdotales y el establecimiento de alianzas son también elementos frecuentes. Un buen ejemplo es el relato sobre el ascenso al poder de Hunac Ceel en el Chilam Balam de Chumayel, quien tiene que pasar por un rito iniciático (arrojarse al cenote y salir con la palabra; es decir, morir ritualmente y renacer estableciendo comunicación con las deidades) para transformarse finalmente en ahau, vínculo con lo sobrenatural y finalmente ser reconocido como halach uinic, representante de los valores tradicionales entre los hombres. Otro ejemplo de discurso de legitimación es la pintura del árbol genealógico de los Xiu, que contiene elementos que aluden a simbolismos prehispánicos. Entre ellos destaca el origen del árbol genealógico, que se equipara con el árbol sagrado o imix che, ya que se sitúa al centro de otros 4 árboles y está enraizado en la cadera de Hun Uitzil Chac, ancestro fundador del linaje Xiu (Okoshi, 2000: 227-231). En este documento se realza su vínculo matrimonial con una señora de Ticul (Ix Ticul). Los relatos sobre el origen del primer Na Mo Ch'el (gobernante de Ah Kin Ch'el) son similares y replican muchos elementos utilizados por los Xiu (Pérez, 2009: 293-323).
} 


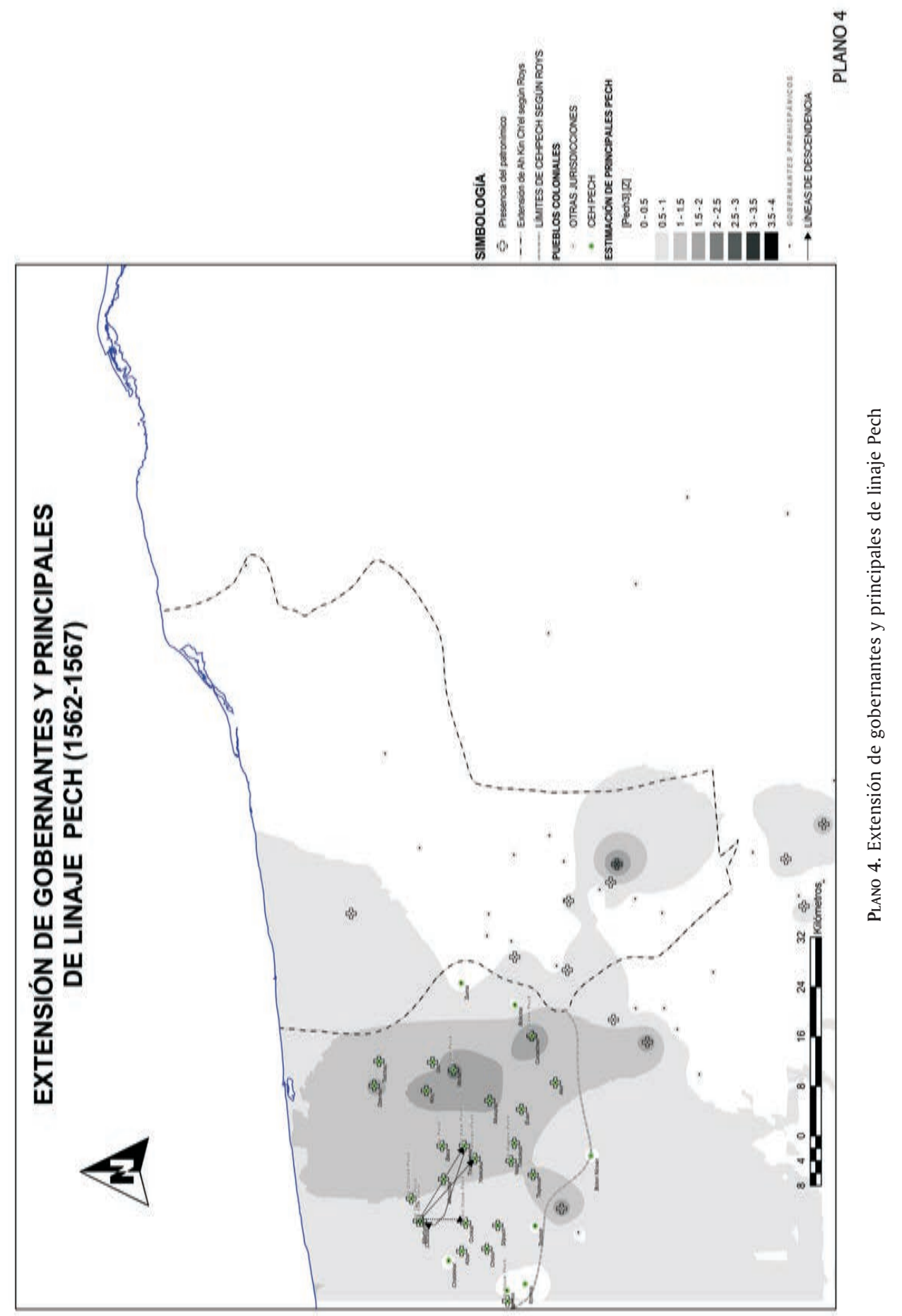


de 25 casos analizados no corresponden a individuos Pech. ${ }^{20}$ Asimismo, a nivel de indígenas principales, tampoco se observan asociaciones conspicuas en sus cabildos, lo que incluso indica que, dentro de cada pueblo, los Pech controlaban la interacción de los miembros de la nobleza, evitando la formación de alianzas entre los otros linajes y cualquier riesgo para el control del grupo que regía su cuchcabal.

El origen de los Pech como linaje gobernante, de acuerdo con las frecuencias y distribución observada, alude a un origen local. La etimología maya literalmente significa "garrapata" y también "aplastar o machucar" (Diccionario Maya, 2001: 642-643). Algunos documentos coloniales asignan un origen foráneo a los Pech, pero la referencia es vaga, "del oriente" y se entrelaza con el surgimiento de Mayapán. ${ }^{21}$ Curiosamente, los documentos escritos directamente por los Pech, como la Crónica de Chacxulubchen y la Crónica de Yaxkukul, omiten el origen de su señorío, ${ }^{22}$ un patrón típico de los nobles yucatecos que se asumen como señores naturales y, por tanto, no expresan en sus documentos el origen de su linaje y su derecho a gobernar.

\section{Las alianzas de los Cupul}

El caso de los Cupul es peculiar, ya que al parecer no había un gobernante supremo ni tampoco una unidad política central. No obstante, la abundancia de personajes del linaje Cupul gobernando distintos pueblos vecinos sugiere algún nivel de organización regional. Roys clasificaba a Cupul y Ah Canul dentro de su segunda forma de organización territorial, donde, en lugar de un gobernante único, había una gran cantidad de batabob pertenecientes a un mismo linaje (Roys, 1957: 6-7). Por su parte, Quezada ubica tres diferentes cuchcabalob dentro de este territorio (Chichén Itzá, Ek Balam y Sací) y varios batabiloob no agregados (como Dzitás, Ebtún, Mexcitán, Yalcobá y Yalsihon) (Quezada, 1993: 160-182).

\footnotetext{
${ }^{20}$ Considerando toda la extensión de Cehpech. Las únicas excepciones son Juan Ek y Gaspar Oxte, gobernadores de Suma y Bokobá respectivamente.

21 "El primer señor de este pueblo de Mutul tienen noticia los naturales de él que fue un Capitán que se llamaba Zacmutul [Sac Mutul], que quiere decir hombre blanco, como tenemos referido. Este vino con gente de hacia la parte de oriente a buscar dónde poblar, y no saben de dónde salió, pero que era indio. Este llegó al asiento donde este pueblo está y le pobló con su gente, y allí hizo su habitación y morada, y tuvieron el señorío él y sus descendientes ciento y cuarenta años, al cabo de los cuales vino contra el señor que entonces era del dicho pueblo de Mutul [Motul], otro señor y Capitán llamado Kaku Pacal [Kak u Pacal], con gente de guerra y le mató y despobló el pueblo. Y al cabo de muchos años, otro señor y Capitán llamado Noh Cabal Peche [Nohcabal Pech], pariente muy cercano del gran señor de Mayapán, tornó a poblar con gente que consigo trajo el dicho pueblo de Motul, y desde entonces tuvieron el señorío este Noh Cabal Peche [Nohcabal Pech] y sus descendientes. Y el día de hoy es cacique y Gobernador del dicho pueblo don Juan Peche [Pech], descendiente de los Peches [Pech], antiguos” (RHGGY, 1983, I: 269-270).

${ }^{22}$ Entendido simplemente como el "dominio o mando sobre alguna cosa, como propia o sujeta" (Diccionario de Autoridades, 2002 [1732), v. 2: 89).
} 
Más precisa nos parece la interpretación de Okoshi, quien señala que Cupul estaba integrado por al menos cuatro cuchcabalob gobernados por diferentes batabob pertenecientes al linaje Cupul: Chichén Itzá (que tenía como sujetos a Sodzil, Tecay y Tikuch); Sací (cuyos sujetos eran Dzitnup, Pixoy, Sisal y Tizimín); Ekbalam (que tenía como sujetos a Hunabkú, Nabalam, Tahcabo y Yalcobá); y Popolá (que tenía trece batabilob sujetos). Asimismo aporta evidencia en el sentido de que, cuando se veían afectados los intereses de estos cuchcabalob (como cuando aconteció la conquista española), los Cupul se unificaban bajo el liderazgo del gobernante de Sací (Okoshi, 1997: 3-6). De cualquier manera, esta situación refleja un arreglo diferente donde la importancia política de un linaje trasciende la esfera de un solo cuchcabal.

Nuestro análisis espacial refleja esa situación. Sea o no Cupul una entidad política o bien un conjunto de ellas, los Cupul se encontraban circunscritos casi totalmente a los poblados del extenso territorio definido por Roys. Se encontraron pocas asociaciones espaciales significativas con otros patronímicos. Uno de ellos son los Uc, que presentan una tendencia de distribución de este a oeste.

Del número de pueblos que se consideran como pertenecientes a esta jurisdicción, la mayor parte se encontraban gobernados por personajes de apellido Cupul -indicativo de su influencia política-, a pesar de que no estaban integrados en una cabecera única, sino por un grupo de batabob que reconocían a uno de su linaje como una cabeza representativa. Si bien ocurren una gran cantidad de patronímicos diferentes en los cabildos indígenas de los pueblos de Cupul, las repeticiones son pocas y no presentan asociaciones espaciales consistentes (planos 5 y 6).

En diversos documentos coloniales, los Cupul se asumen como "señores naturales"; o bien, no mencionan su origen y la legitimidad como gobernantes. El significado del apellido está vinculado con la palabra maya para la jícama cimarrona (Calopogonium coeruleum) (Diccionario Maya, 2001: 354). ${ }^{23}$ En las Relaciones histórico geográficas de la gobernación de Yucatán existen breves menciones sobre su carácter local (RHGGY, 1983 II: 267). Es muy sugerente la mención de la Relación de Valladolid que refiere que la gente de Chikinchel llamaba despectivamente a los pobladores de Cupul como "ah maya". ${ }^{24}$ Solamente la Probanza de Don Juan Kauil, documento de principios del siglo xvir, relaciona a los Cupul con México y Chichén Itzá. ${ }^{25}$

${ }^{23}$ Alfredo Barrera Vásquez han sugerido que cup es posiblemente un nahuatlismo, aunque no da mayor información (Ibid: 353).

24 “...llamaban antiguamente los indios de la provincia de Chiquinchel [Chikinchel] a éstos de esta villa de Valladolid, y las demás provincias de los copules [Cupul] y Cochua [Cochuah], ah mayas, ultrajándolos de gente soez y baja, de viles entendimientos e inclinaciones" (RHGGY, 1983, II: 37).

${ }^{25}$ La Probanza de Don Juan Kauil de 1618 señala un origen foráneo de los Cupul: México y Chichén Itzá. Asimismo, asocia a uno de los ancestros como pariente de Moctezuma (en Brinton, 1969 [1882]: 114-116). 


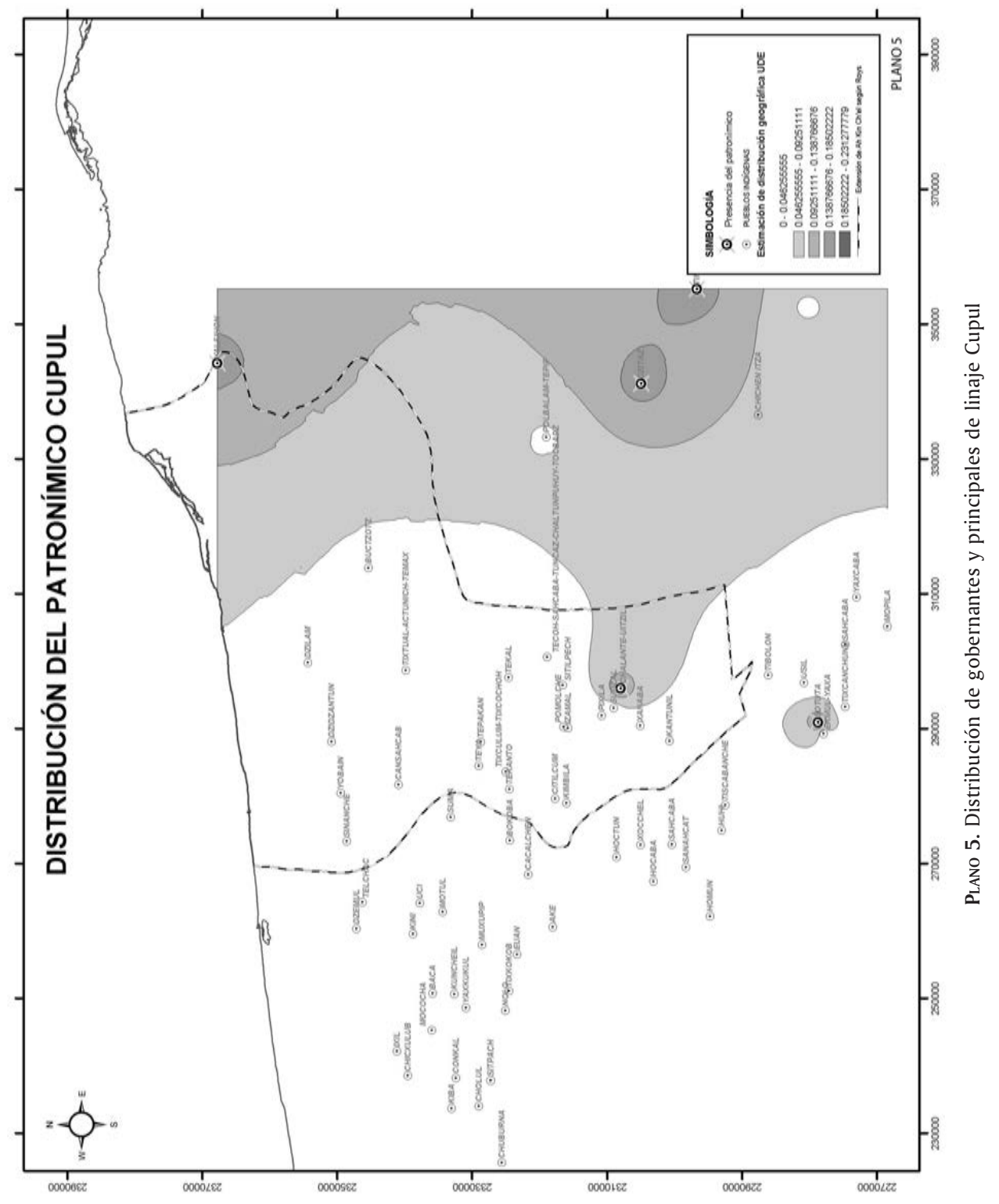




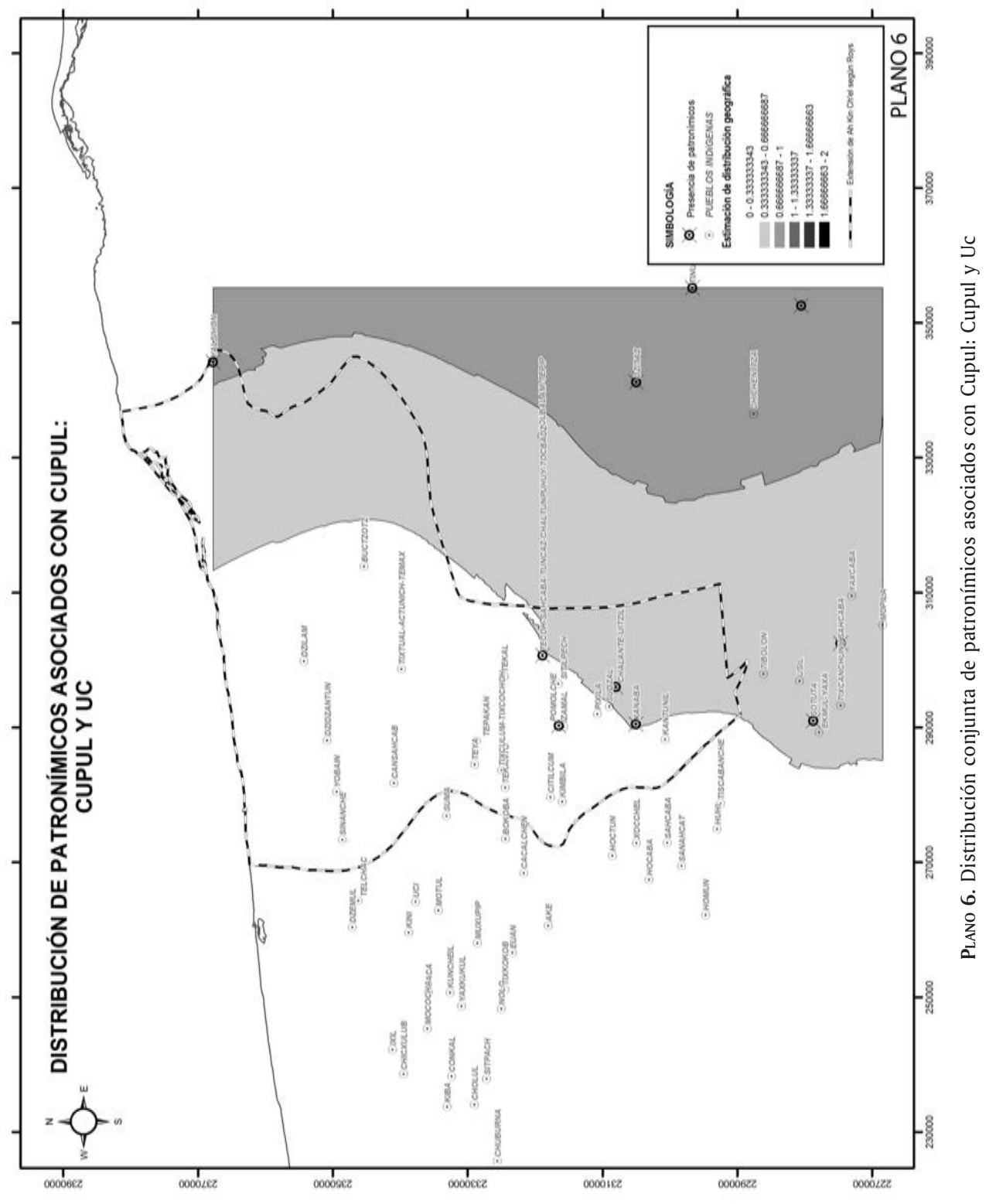




\section{Las alianzas de los Ch'el}

Los Ch'el, a pesar de su escaso número, establecieron una intrincada red de relaciones y vínculos en los pueblos de su cuchcabal. A diferencia de sus vecinos de Ceh Pech, Cupul, Hocabá y Sotuta, los miembros de su linaje no ocuparon la mayor parte de las posiciones de gobierno. Las alianzas mantuvieron a los linajes locales acotados y fragmentados de manera que no constituyeran un riesgo a su papel preeminente en el cuchcabal. Miembros de linajes como los Camal, Motul, Mex, Dzib, Cauich, Cahun, Che y Mocul fueron parte medular de la estructura política (planos 7-9). Al interior de los pueblos, el poder político fue compartido con batabob de 26 distintos linajes, destacando por número personajes Chan, Motul, Tun, y Ucan e incluso miembros de linajes rivales como Pech y Cupul en áreas fronterizas. Los datos arqueológicos permiten suponer que durante el Posclásico existieron entre 31 y 37 batabilob dependientes de los señores Ch'el.

En los dos casos donde personajes Pech figuran como gobernadores de pueblos dentro de Ah Kin Ch'el, son fronterizos con la jurisdicción de Cehpech (Uitzil y Kimbilá), justamente en el área donde, se asume, pudo haber algún tipo de pugna en época prehispánica. Se tienen dos posibilidades de una rivalidad política. Primero, que dichos pueblos no pertenecían realmente a la jurisdicción de Ah Kin Ch'el. El segundo, y más probable, que esos dichos pueblos originalmente pertenecían a los Pech y su subordinación a los Ch'el pudo originarse por un conflicto militar; o bien, una escisión política de los Pech de Uitzi y Kimbilá con Cehpech y una renegociación política con los nobles Ch'el. Recordemos las frecuentes menciones en las relaciones histórico geográficas de la Gobernación de Yucatán sobre la enemistad entre los Ch'el y los Pech.

Los Cupul en Ah Kin Ch'el se encuentran igualmente en pueblos fronterizos y con adscripción política dudosa, Chalamté y Yasilhón. Se puede inferir que las relaciones de los Ch'el con los distintos pueblos de Cupul eran diferenciales y dinámicas, lo cual concuerda con la aparente inexistencia de un poder unificado en Cupul. En consecuencia, las afinidades y diferencias se establecerían localmente de acuerdo con la situación imperante. Con los pueblos de la zona sur de Cupul aparentemente no existían conflictos (Chalamté). Sin embargo, en la región norte y fronteriza con Ah Kin Ch'el (Yasilhon), la situación era hostil. Una posible explicación para este conflicto sería el control de las bocas de Dzilam, punto estratégico de acceso a la costa, tanto para los Cupul como para los Ch'el. En el área oriente de Ah Kin Ch'el hay indicios de conflictos armados (entre Tepakan y Chocholá). ${ }^{26}$

\footnotetext{
${ }^{26}$ Como ejemplo de lo anterior, la relación de Kampokolché y Chocholá, refiere los conflictos que sostenía este último pueblo, situado al oriente de Tizimín, con el Nacom Yok de Tepakan, dentro de Ah Kin Ch'el. Asimismo, el encomendero Giraldo Díaz de Alpuche, encomendero de Dzonot, refiere los conflictos de este pueblo con Buctzotz y Dzilam: Este pueblo, a lo que me dijeron dos viejos que hay en él, reconocía a tres principales, el uno se llamaba en su lengua batab, que quiere decir en nuestra lengua Capitán, a éste no le tributaban nada, más que se recogían en su casa a tratar
} 


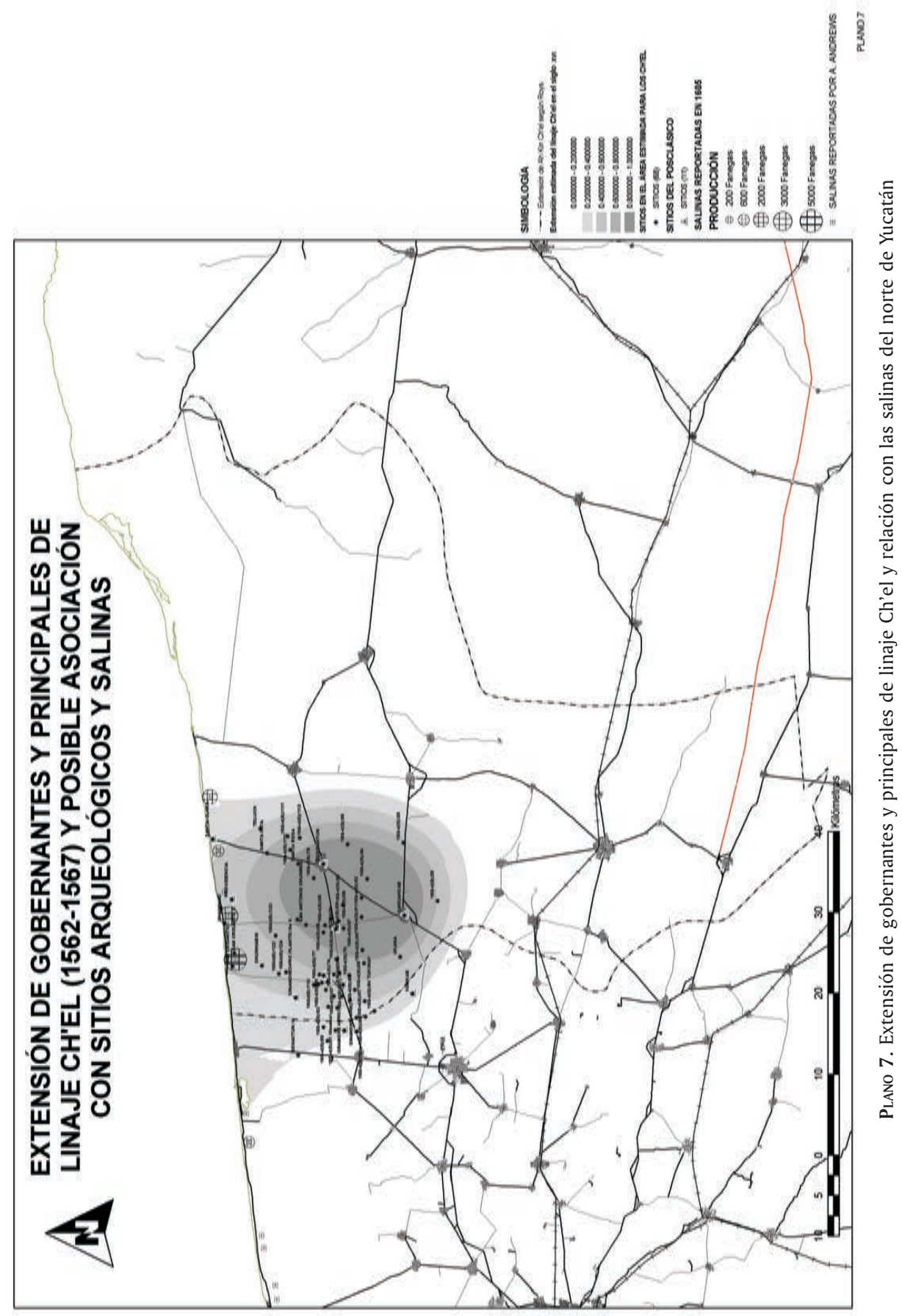




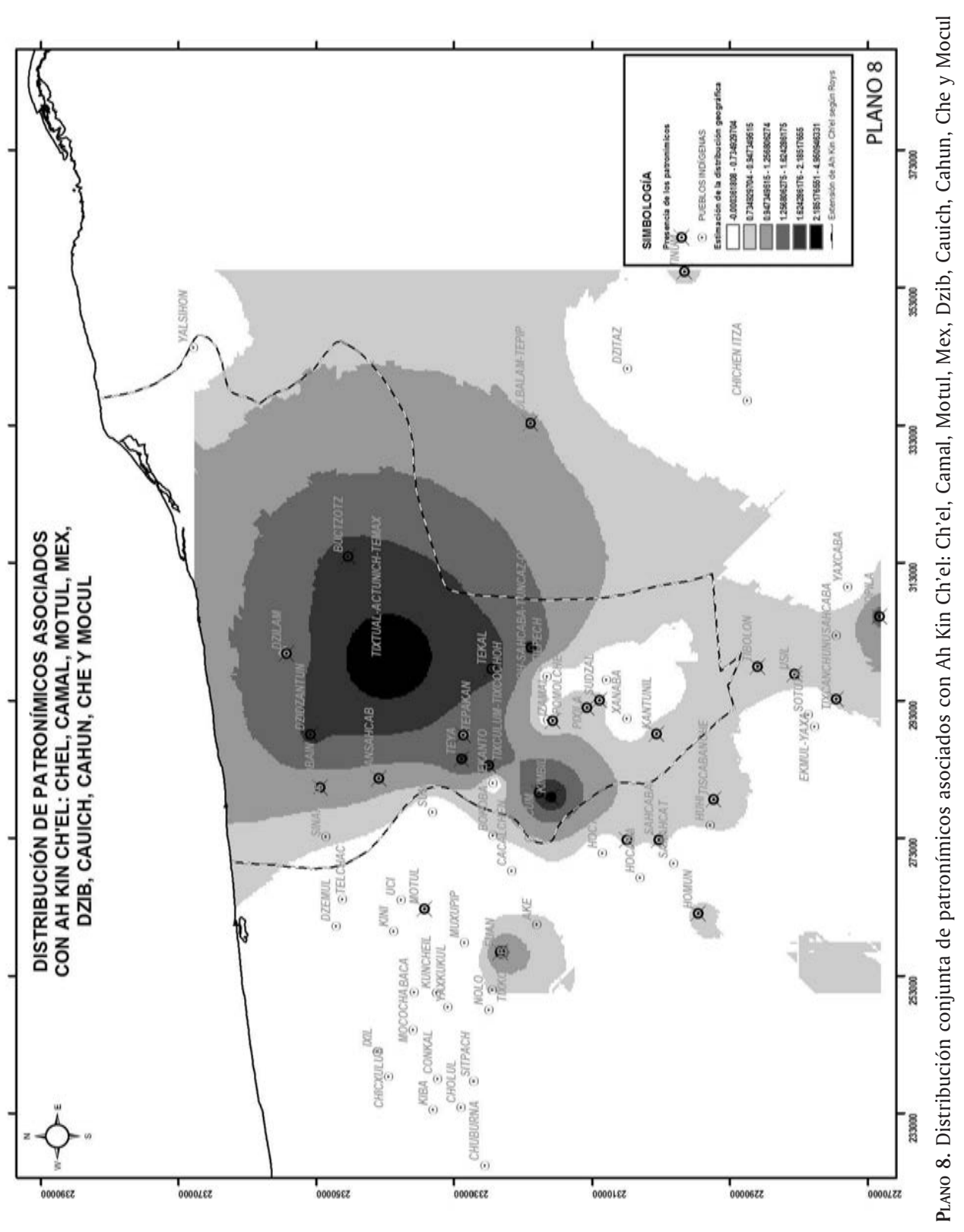



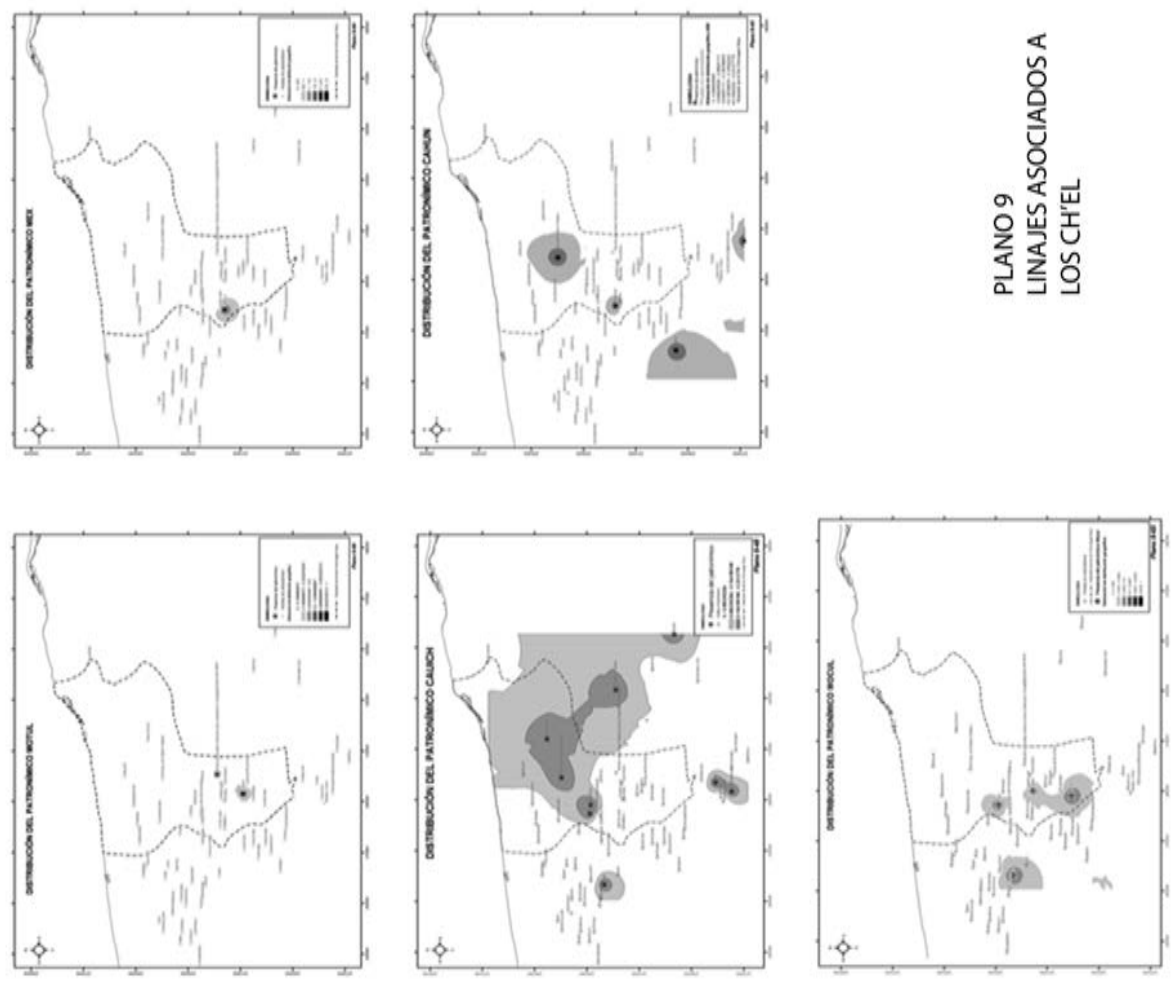

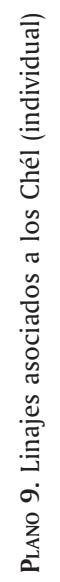
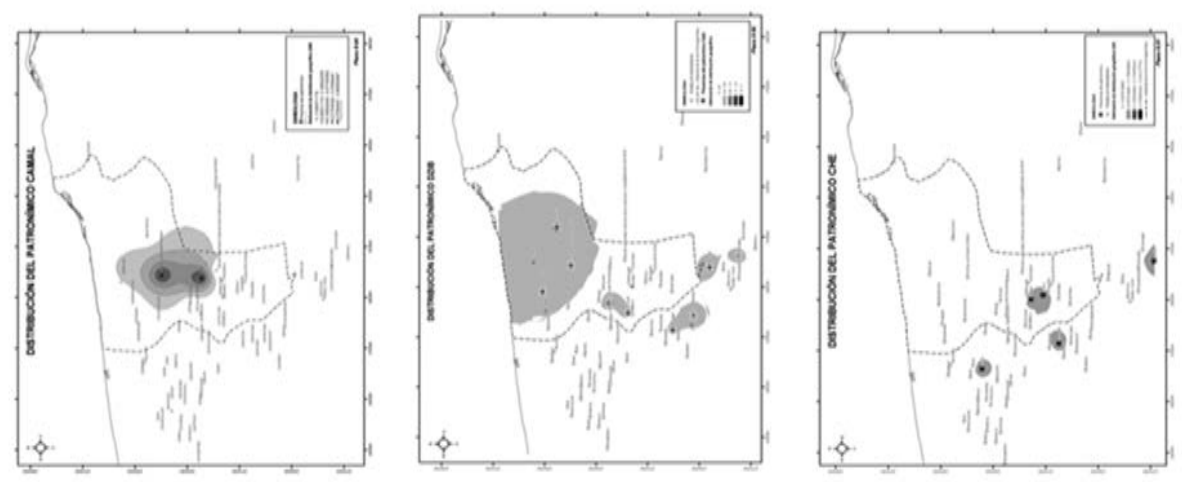
Con respecto a Hocabá y Sotuta, las relaciones parecen haber sido más estrechas debido a que son más los casos de linajes asociados espacialmente con Ah Kin Ch'el, Sotuta y Hocabá, que con respecto a Cehpech y Cupul. Si bien hay una notoria exclusión de los Cocom en los pueblos de Ah Kin Ch'el, ambos cuchcabalob comparten patronímicos que se ubican en pueblos de ambas jurisdicciones: Euan, Uicab y Balam. Esto sugiere que la pretendida enemistad entre Ch'el y Cocom no era tan acentuada y que existía un contacto entre los nobles locales de ambas jurisdicciones. Entre Hocabá y Ah Kin Ch'el se han documentado contactos e intercambio de "presentes" entre los gobernantes de los pueblos de cada una de esta jurisdicciones (Roys, 1957: 82). La evidencia apunta a que Hocabá sostenía buenas relaciones tanto con Ah Kin Ch'el como con Sotuta. Es relevante que, de los tres linajes que se asocian con estas tres jurisdicciones (Chan, Baz y Tun), dos tienen más poder político en el sur de Ah Kin Ch'el a juzgar por el número de gobernadores de pueblos (Chan y Tun).

Puede caracterizarse a los Ch'el como un linaje minoritario, foráneo y recién establecido, que requirió de múltiples alianzas y arreglos políticos para afirmar el dominio de sus pueblos y de los recursos. Este esquema es consistente con los relatos históricos sobre su origen y legitimidad. Na Mo Ch'el, sacerdote o $A h \mathrm{Kin}^{27}$ de linaje paterno Ch'el, se convirtió en el gobernante principal o halach uinic de este cuchcabal. Fue discípulo y yerno de Ah Xupan Xiu, un importante sacerdote y/o gobernante de Mayapán.

El nombre Chel o Ch'el hace alusión a una ave caracterizada por el tono negro azulado de su plumaje, la urraca yucateca (Cissolopha yucatanica) ${ }^{28}$ La iridiscencia del plumaje de estas aves y el uso moderno de ch'el como calificativo moderno de personas rubias, posiblemente esté relacionado con la voz chel o cheel que significa "arco del cielo" o "arcoíris," y constituye parte del nombre de la diosa lunar Ix Chel..$^{29}$ El patronímico Ch'el es reportado por Roys como muy escaso en la península y restringido al oeste de Yucatán (Roys, 1940: 35).

algunos negocios y a holgarse, y cuando salían a alguna guerra lo llevaban como por cabeza porque éstos tenían guerra con una provincia que se llamaba Kinchel [Ahkinchel], que la cabecera se llama Tabuctzotz [Buctzotz] y Silam [Dzilam] ( ${ }_{R} H G G Y, 1983$ II: 85). Es interesante que la descripción de este encomendero difiere de la concepción generalizada de las atribuciones del batab.

${ }^{27}$ Ah Kin es el sustantivo maya para sacerdote. Literalmente puede traducirse como "el del Sol" o "el del día". Thompson (1984: 213) considera que el significado de kin en este contexto es el de adivinación. Así, pone de ejemplo las palabras tzac kin (hechizo) y kinyah o kintah (pronosticar) determinativo. Sin embargo, reconoce que la derivación original probablemente venga del significado de kin como "día", ya que los pronósticos de los sacerdotes se basaban en el estudio de la suerte de los días.

${ }^{28}$ Calepino de Motul, 1995, I: 261. El Diccionario Maya (2001: 131) tiene las siguientes acepciones: pájaro especie de arrendajos; arrendajo o ave negra, de plumas azules; urraca Cyanocitta yucatanica; pájaro azul; la urraca yucateca Cissolopha yucatanica. Otra entrada asienta ch'el como adjetivo para pelirrubio o persona de tez blanca y pelo claro. Asimismo, ch'eel, con vocal larga, significa cimiento o asiento que se hace del edificio y su sinónimo en maya sería dzamal.

${ }^{29}$ Calepino Maya de Motul, 2001: 194. Ver también el trabajo de Noemí Cruz (2005: 14-16) sobre las voces relacionadas con chel. 
Los Ch'el, constituyeron un linaje de cierta preeminencia dentro de la estructura de poder de Mayapán. Es posible que conformaran parte de las diferentes migraciones que se asentaron en Yucatán a finales del Clásico Terminal. Su vínculo con los Xiu es innegable y es probable que tomaran parte en las pugnas de poder que finalmente terminaron con la destrucción del asentamiento de Mayapán y la diáspora de los distintos grupos de la nobleza indígena hacia áreas más propicias para reconstituirse.

Los Ch'el se asentaron en el centro norte de la península yucateca por diversas razones. En primer lugar, el aprovechamiento de una serie de recursos costeros para la subsistencia y el intercambio de bienes: sal y pescado. En segundo, un área fragmentada políticamente y donde linajes locales fuertes como los Pech y los Cupul aún no conseguían un dominio estable (plano 10).

Los documentos que más datos nos aportan sobre el origen del linaje Ch'el son la Relación de las Cosas de Yucatán de Fray Diego de Landa y el conjunto de documentos conocido como las Relaciones Histórico Geográficas de la Gobernación de Yucatán..$^{30}$ A pesar de haber sido elaborados en español y con un formato occidental, ${ }^{31}$ se advierte la participación de informantes indígenas, destacando en ambos casos el célebre Gaspar Antonio Chi Xiu, intérprete de indios del cabildo de Mérida. Asimismo, una comparación detallada con algunos fragmentos de los relatos indígenas, revela reminiscentes de la estructura discursiva típica de los textos y narraciones orales de los mayas. El discurso de legitimación de los Ch'el conduce a deducir el proceso de constitución del cuchcabal. Un primer establecimiento cercano a la costa, tomando control directo de pueblos como Dzidzantún, Yobaín, Cansahcab y Dzilam. Posteriormente, se da la consolidación de su poder a través del establecimiento de una capital en Tecoh, reutilizando asentamientos y la posición geográfica de la antigua área de influencia de Izamal, para tener acceso a tierras más fértiles y rutas de comunicación hacia el interior de la península. Este proceso coincide con el reconocimiento definitivo del primer Ah Kin Ch'el como ahau y halach uinic de esta región, tras un "reconocimiento" por parte de los Cupul.

En el análisis de las narraciones sobre el acceso al poder de Ah Kin Ch'el se detectaron varios procesos que muestran, por un lado, un reconocimiento y un rescate de la visión tradicional sobre la legitimación de los gobernantes, y, por

30 Relación de Cansahcab (RHGGY, 1983, I: 94-95) y Relación de Izamal y Santa María (RHGGY, 1983, I: 304-305).

${ }^{31}$ Recuérdese que, más que un desinteresado afán de dejar constancia escrita de la vida e historia de los mayas yucatecos antes de la conquista, la relación que escribió fray Diego de Landa tuvo como principal propósito justificar, ante la corona española, sus actos como provincial de los franciscanos en el llamado auto de fe de Maní de 1562, por el cual tuvo que comparecer en España. No obstante, en el resumen de su obra que ha llegado hasta nosotros, es perceptible cierto rigor al contrastar los datos y las versiones ofrecidas por sus informantes indígenas. Por su parte, las Relaciones Histórico Geográficas de la Gobernación de Yucatán obedecen a un requerimiento administrativo de la corona española solicitado a través de un cuestionario información sobre las posesiones españolas en ultramar. 


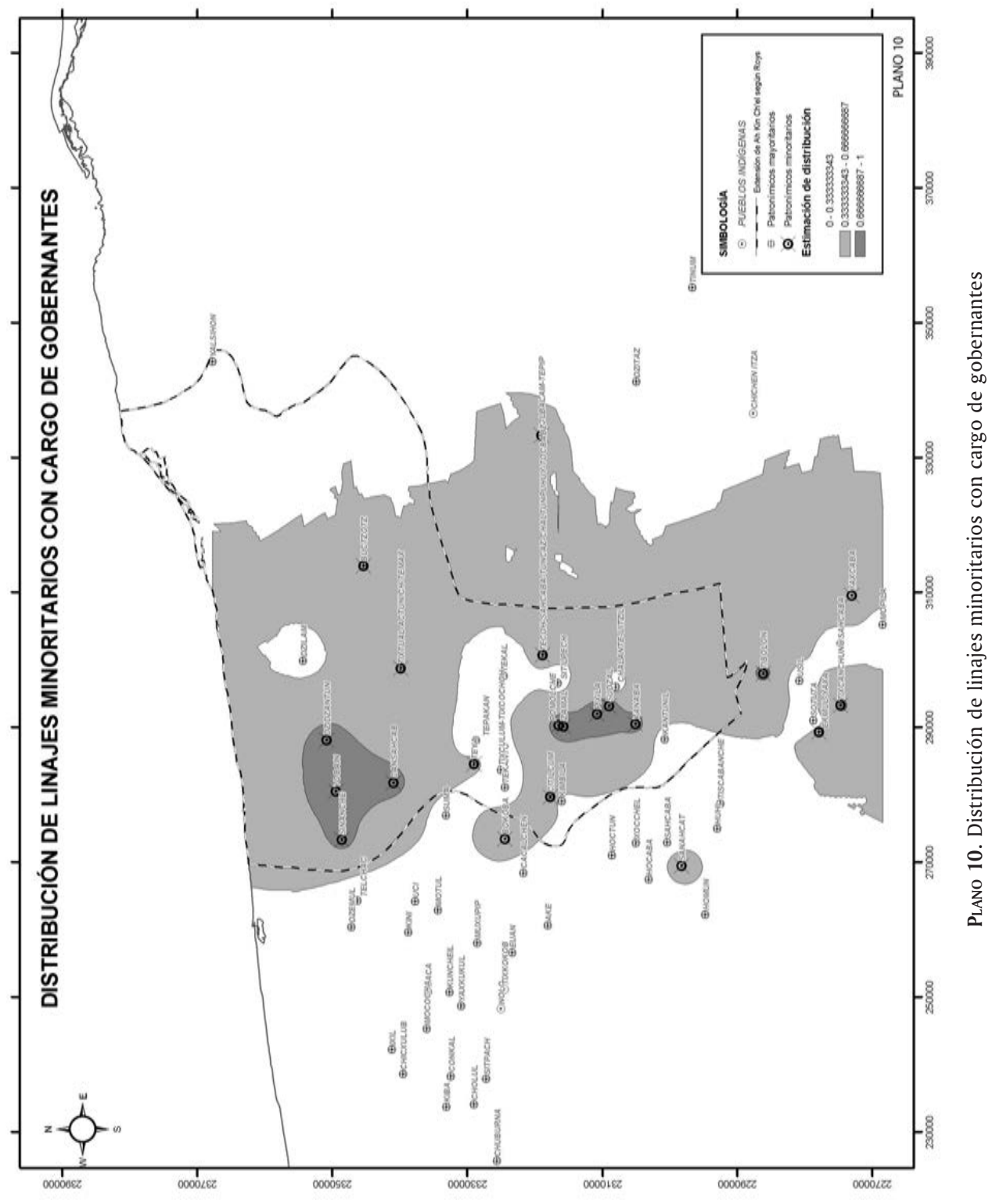


otro, una adaptación a los nuevos esquemas del poder político y las formas de gobernar.

De manera similar a Hunac Ceel de Mayapán, el primer Na Mo Ch'el no tuvo en principio un derecho legítimo a gobernar y su validación se realizó a través de alianzas matrimoniales y el realce de sus cualidades como Ah Kin o sacerdote. Es decir, el conocimiento de los rituales religiosos, la lectura de los libros antiguos y las artes de la adivinación. Esto fundamenta su reconocimiento como un ahau, un enlace entre hombres y deidades.

Las fuentes refieren que el primer Na Mo Ch'el se casó con la hija del sacerdote de Mayapán, Ah Xupan Xiu, otras lo refieren como su "criado", de manera similar que Hunac Ceel Cauich con Ah Mex Cuc. Ambas situaciones no se contraponen. La costumbre del yerno de servir en la casa de su suegro un par de años se mantuvo hasta mediados del siglo pasado en Yucatán.

Esta estrategia de ofrecerse en servicio y enlazarse con mujeres nobles está documentada en varios casos del centro de México como los mexica en Culhuacan (Durán, 1995 Cap. IV: 84).

Finalmente, los Ch'el tenían que justificar su reconocimiento como gobernantes justos de acuerdo con los parámetros del Posclásico. Con este fin, su discurso histórico retomó las cualidades de personajes como Quetzalcóatl y Tutul Xiu. La facultad de negociar alianzas y validar acuerdos, de gobernar "más por maña que por fuerza", fue la base para su reconocimiento como halach uinic, como verdaderos y legítimos gobernantes.

\section{Las alianzas de los Iuit}

Los luit constituyeron un linaje restringido al cuchcabal que gobernaban, Hocabá. Su dominio político era más sólido comparativamente con los Ch'el y los Cocom, pues cinco de ocho pueblos principales del cuchcabal eran gobernados por personajes luit (Xocchel, Hocabá, Hoctún, Tiscabanchel y Huhí). Los Noh constituyen el único linaje asociado de manera significativa con los luit, lo cual indica que los vínculos políticos directos eran más fuertes al interior del linaje luit, destinados a controlar a los pueblos del cuchcabal de Hocabá. ${ }^{32}$ Las asociaciones espaciales de otros patronímicos compartidos con Ah Kin Ch'el y Sotuta, probablemente estén relacionadas con los contactos diplomáticos que sostenían los principales de Hocabá con sus vecinos (planos 11 y 12). ${ }^{33}$

\footnotetext{
${ }^{32}$ En la Relación de Valladolid se refiere la existencia de un capitán llamado Na Cahun Noh que residía en Valladolid y al cual temían y ofrendaban los cupules de la provincia de Tizimín (RHGGY, Relación de la Villa de Valladolid, 1983, T.II: 28)

${ }^{33}$ Autores como Roys han señalado la "buena” relación entre los cuchcabalob de Hocabá y Ah Kin Ch'el durante el Posclásico. En las indagaciones sobre el auto de fe de Maní se registra el hecho de que el batab de Cansahcab (del linaje Ch'el) envió como regalo al batab de Huhí seis doncellas para ser sacrificadas: "Y que el mismo Lorenzo luit decía a los demás caciques que estas muchachas las
} 
El origen de los luit no parece local. Al igual que los Ch'el, Roys refiere que se trata de apellidos poco frecuentes con una distribución limitada en el oeste de Yucatán. Por su parte, entre los chontales de Tixchel, los luit sí constituyeron un nombre relativamente frecuente (Roys, 1940: 36).

El origen etimológico apoya lo anterior, ya que iuit parece provenir del náhuatl iuitl o yhuitl, que significa "pluma menuda" (Molina, 1992: 96v; 44r). En su versión náhuatl o castellanizada (pluma), este apellido se encuentra aún muy difundido en algunas partes del centro de México, tales como la región de PueblaTlaxcala.

\section{Las alianzas de los Cocom}

Según los datos, los Cocom gobernaban de manera directa 3 de 10 pueblos de su cuchcabal (Sotuta, Ekmul y Sahcaba). En los otros pueblos de Sotuta, se observa un patrón peculiar, ya que estaban gobernados por personajes cuyos patronímicos son muy escasos y prácticamente restringidos a Sotuta (Ix, Tuyú, Hau, Nagual).

Los Cocom muestran grandes similitudes con los Ch'el en el comportamiento de los linajes de principales asociados, lo cual puede referir estrategias similares para consolidar el poder en una región determinada. Los Cocom eran más abundantes y más concentrados espacialmente. No obstante, también se vincularon con otros linajes nobles, a semejanza del caso de los Ch'el. Ambos son linajes numéricamente escasos, pero dominantes políticamente a nivel local, que aprovecharon los vínculos con linajes de papel secundario, pero con una distribución geográfica consistente (Euan, Uicab, Balam, Canul, Can, Couoh, Zulú, Chan, Baz y Tun (planos 13-15).

El caso de los Cocom es paradójico, la distribución y etimología del apellido apuntan a un posible origen foráneo; sin embargo, en los relatos históricos, el esquema de legitimación de los señores Cocom es coherente con el de los "señores naturales" o locales.

De acuerdo con las fuentes, los Cocom fueron el linaje que gobernaba Mayapán y que fue derrotado por una rebelión encabezada por los Xiu, lo cual determinó la caída definitiva de Mayapán como centro de poder y casi el exterminio de los nobles Cocom, cuyos sobrevivientes se instalaron en Sotuta. De manera coherente con esta versión, la distribución (plano 13) de la mayoría de los principales Cocom se ubica dentro de los pueblos de este cuchcabal, sólo se nota una extensión hacia Ah Kin Ch'el por la presencia de un Cocom en Tocbadz. Este

\footnotetext{
había enviado el cacique de Cansahcab que se llama Francisco Ch’el, que decían ser de Cah, que ellos llaman la provincia de Izamal, porque así lo tenían de costumbre antiguamente enviarse unos caciques a otros a manera de presentes los muchachos y muchachas para sacrificar que ellos llaman antiguamente quymchich, lo cual se hacía públicamente en los pueblos, y que ahora no lo hacen sino en las milpas en los montes" (Información hecha en el pueblo de Homún sobre la idolatría de los indios. Septiembre de 1562. Residencia de Quijada, AGI, Justicia 249, citado en Scholes y Adams, 1938, I: 136).
} 


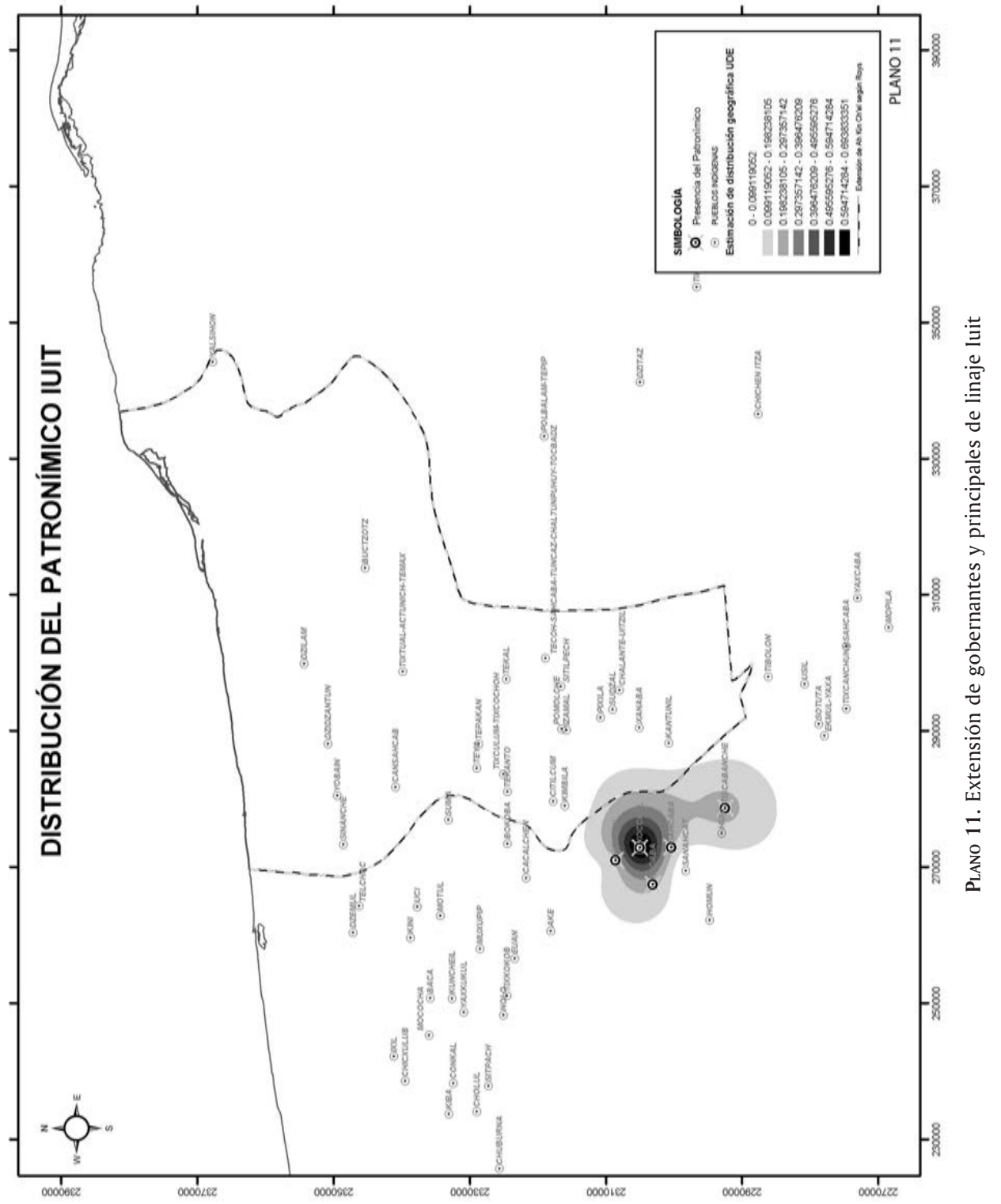




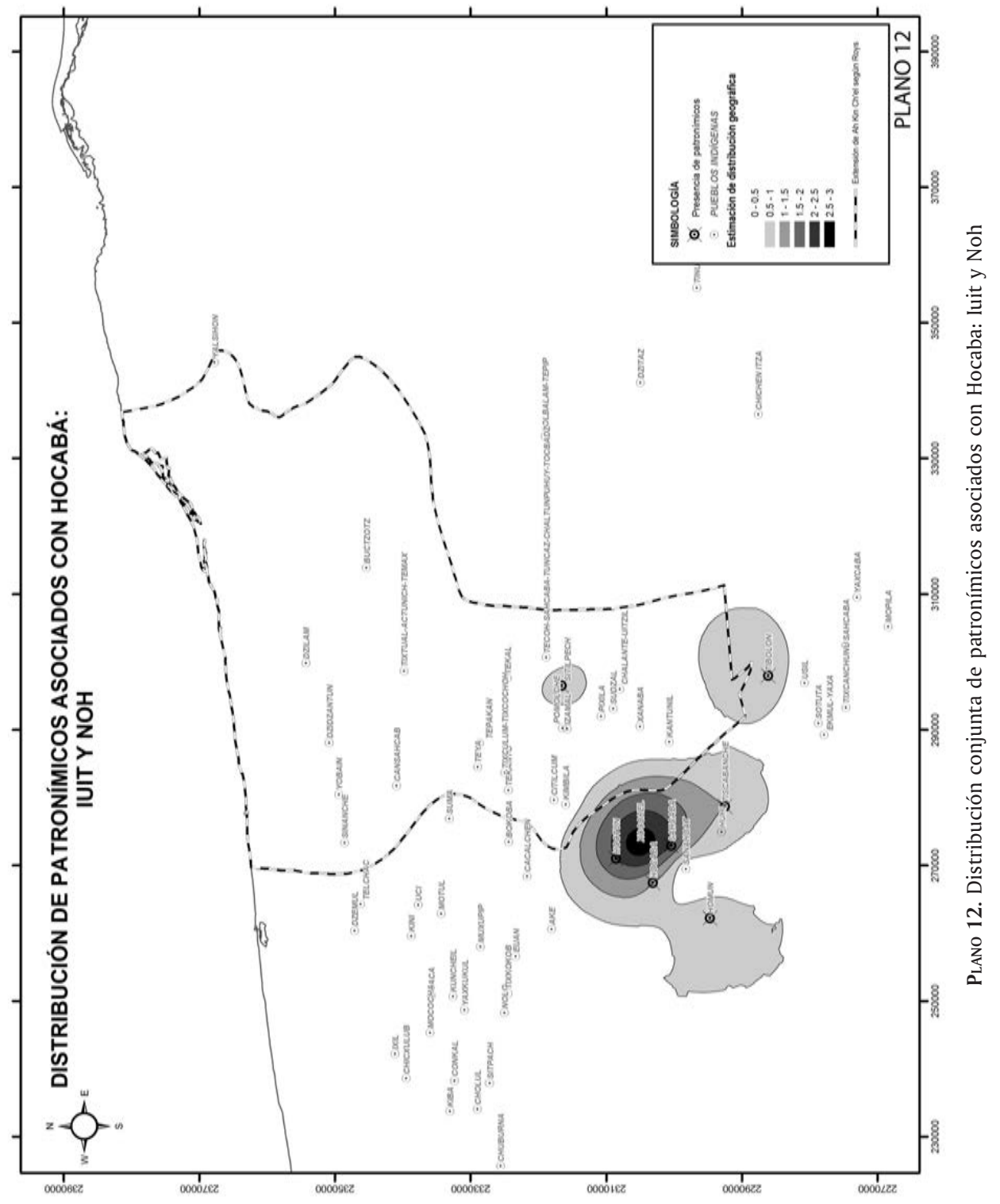


arreglo concuerda con el relato que los señala como un grupo desplazado de su capital política original.

Este movimiento y reacomodo político explicaría el porqué los Cocom muestran un arreglo de tipo concentrado en su nuevo territorio, ubicando a los miembros de su linaje en los pueblos principales cercanos, manteniendo de esta forma su cohesión. Otros datos apoyan esta suposición. Fuera de Sotuta, solamente se reportan batabob Cocom en los pueblos de Tixbecyá, Tecoh y Chaltun, ubicados en la jurisdicción de Chakán, pero cercanos en distancia al asentamiento arqueológico de Mayapán, que, para el Posclásico Tardío, se encontraría dentro del territorio de los pueblos de Maní (Quezada, 1993: 179-180). Por otra parte, analizando a Cocom como apellido común, Ralph Roys, a partir de listas de tributarios de 1584 y 1688, reporta a los Cocom como especialmente abundantes en Hocabá, Sotuta, Cochuah, Cupul y, particularmente escasos, casi ausentes en Maní y Cehpech (con 1 y 2 casos respectivamente).

Al respecto, hay dos posibles conjeturas. La primera, que los Cocom efectivamente fueron desplazados de Mayapán y se refugiaron en Sotuta, reforzando sus lazos políticos con los señores mayas del oriente, caracterizados por sus pautas culturales más conservadoras y su autoasunción como señores naturales. La segunda, en concordancia con las versiones derivadas del discurso de Gaspar Antonio Chi Xiu, de que los Cocom nunca tuvieron un papel protagónico en Mayapán; por consecuencia, su esfera de acción se encontraría en el sur oriente de la península, enlazada con el antiguo auge de Chichén Itzá, y de cuyos vínculos hay indicios en la epigrafía y escritos coloniales tardíos, como la probanza de don Juan Kauil donde se afirma que "le cupo a un Cocom el poblar Chichen Itzá" (Brinton, 1969 [1882]: 114-116).

Los indicios del origen de los Cocom a través de su etimología son escasos y confusos. La acepción de Cocom en el Calepino de Motul figura como "escucha o escuchador con atención". ${ }^{34}$ Los registros más antiguos del vocablo Cocom los encontramos en las inscripciones de Chichén Itzá donde aparece un personaje denominado como K'ul KoKom Yahawal Cho' K'ak (Grube, 1990: 13; Voss y Kremer, 2000: 158). Roys ha sugerido que la voz "cocom" es un participio arcaico en maya que significa "aquél que escucha con atención". Esta evidencia ha llevado a autores como Voss y Kremer a suponer que Cocom fue originalmente un cargo político que derivó en la denominación de un linaje. Sin embargo, no hay muchos sustantivos o verbos con campos semánticos asociados que permitan corroborar esta etimología y la procedencia mayance del apellido. Una alternativa para el origen de este patronímico es su asociación con plantas trepadoras de flores amarillas (coceh, "diente de venado") del género Smilax, conocidas en español como zarzaparrilla (Roys, 1940: 42; 1933: 225-226; Yates, 1994: 16).

${ }^{34}$ Calepino de Motul, 2001: 115. Este significado también es mencionado por Torquemada (1975 [1723], II: 52). 


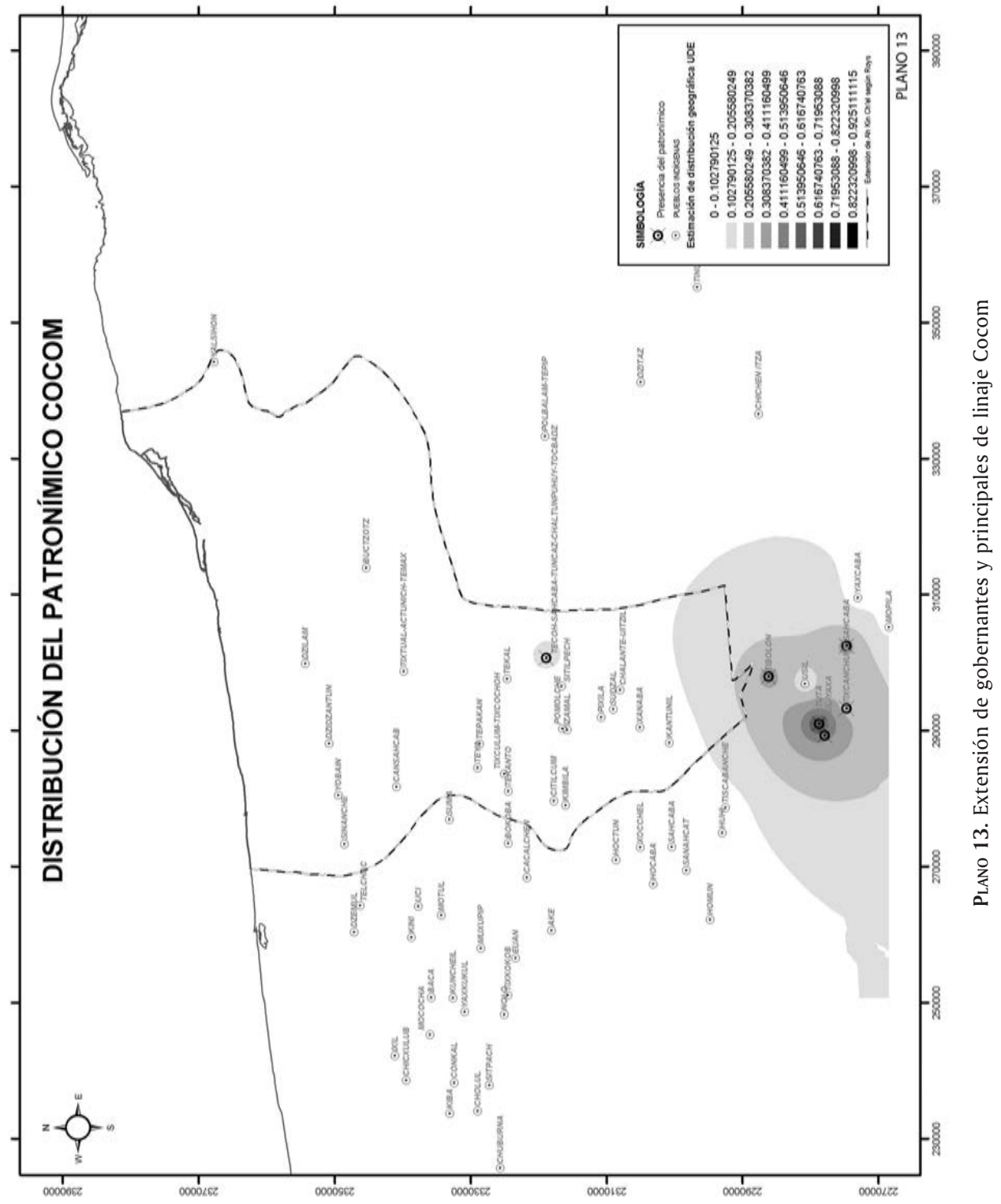




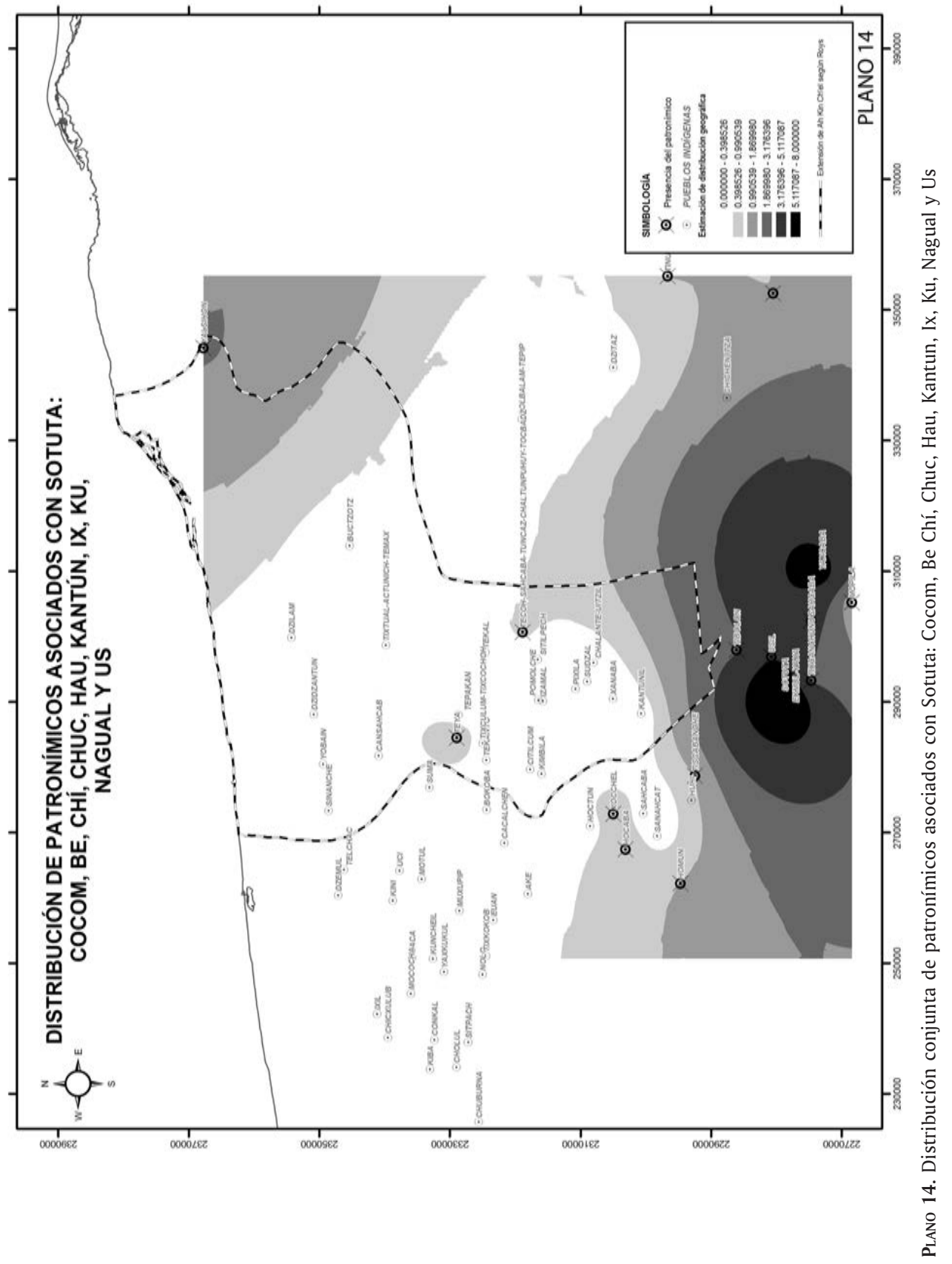


Otra opción interpretativa podría originarse a partir de la lengua náhuatl, asumiendo que la llegada de los Cocom se remontaría a la etapa de Chichén Itzá. Así, cocone es voz utilizada por las mujeres para referirse a sus hijos o hijas pequeñas, tiene relación con el verbo cocomotza, "paladear el niño cuando mama". Cocomoltic es "lugar lleno de barrancos". El verbo coconauia es otra posibilidad, significa "atraer a otro con palabras o dones", y cococaua, "ser señor o dueño de hacienda" (Molina, 1992: 23v). En el ámbito de las plantas, el cocoyome es una cactácea alucinógena que representa una divinidad menor entre los tarahumaras, y el cacolmecatl, el equivalente náhuatl de la zarzaparrilla (Diccionario Maya, 2001: 331). La posibilidad del origen náhuatl se refuerza también por la asociación con el cargo de ah cuat (serpiente), ostentando por Na Chí Cocom en la Crónica de Yaxkukul. ${ }^{35}$

La distribución del patronímico Cocom, ya sea como personajes nobles o de la población general, demuestra una exclusión sistemática de Mayapán y el área controlada por los Xiu. Este argumento es coherente con la versión histórica que supone un desplazamiento geográfico de los Cocom como linaje dominante a la caída de Mayapán y un nuevo proceso de consolidación política en su nuevo asiento, reconstruyéndose ideológicamente a partir de ese momento como señores locales en oposición a sus rivales, los extranjeros Xiu.

\section{Consideraciones finales}

En este trabajo se ha puesto a prueba nuevamente la utilidad de una metodología multidisciplinaria para adentrarnos en el conocimiento de complejos problemas de investigación, como lo es la organización sociopolítica de los antiguos pueblos mesomericanos. Lo que fuera inicialmente una investigación centrada en el cuchcabal Posclásico de Ah Kin Ch'el ha derivado en una investigación a nivel regional con el fin de obtener mayores indicios para determinar el papel de las alianzas políticas entre los linajes nobles de esta época.

La conjunción de análisis de datos y la aproximación al esquema cognoscitivo de los mayas para entender y asimilar los acontecimientos ha permitido elaborar una propuesta reconstructiva de la geografía política de esta porción del norte de Yucatán. Se detectaron patrones distintivos en la distribución y ordenamiento de los linajes nobles que guardan correspondencia con la estructura organizativa particular de cinco jurisdicciones estudiadas. Por un lado, tenemos dos linajes de tradición local, Pech y Cupul, que muestran un patrón de amplia dispersión, con pocas alianzas con linajes secundarios para establecer el control de sus pueblos, lo que da indicios de una estructura política dominante basada en los lazos de sangre. En el caso de los Pech, la estructura de gobierno estaba centralizada,

${ }^{35}$ Crónica de Yaxkukul, 1574, f.13; 26 Yucatan Collection Box 1 Folder 3,4,5,6,7, Tulane University, Howard Tilton Memorial Library. 

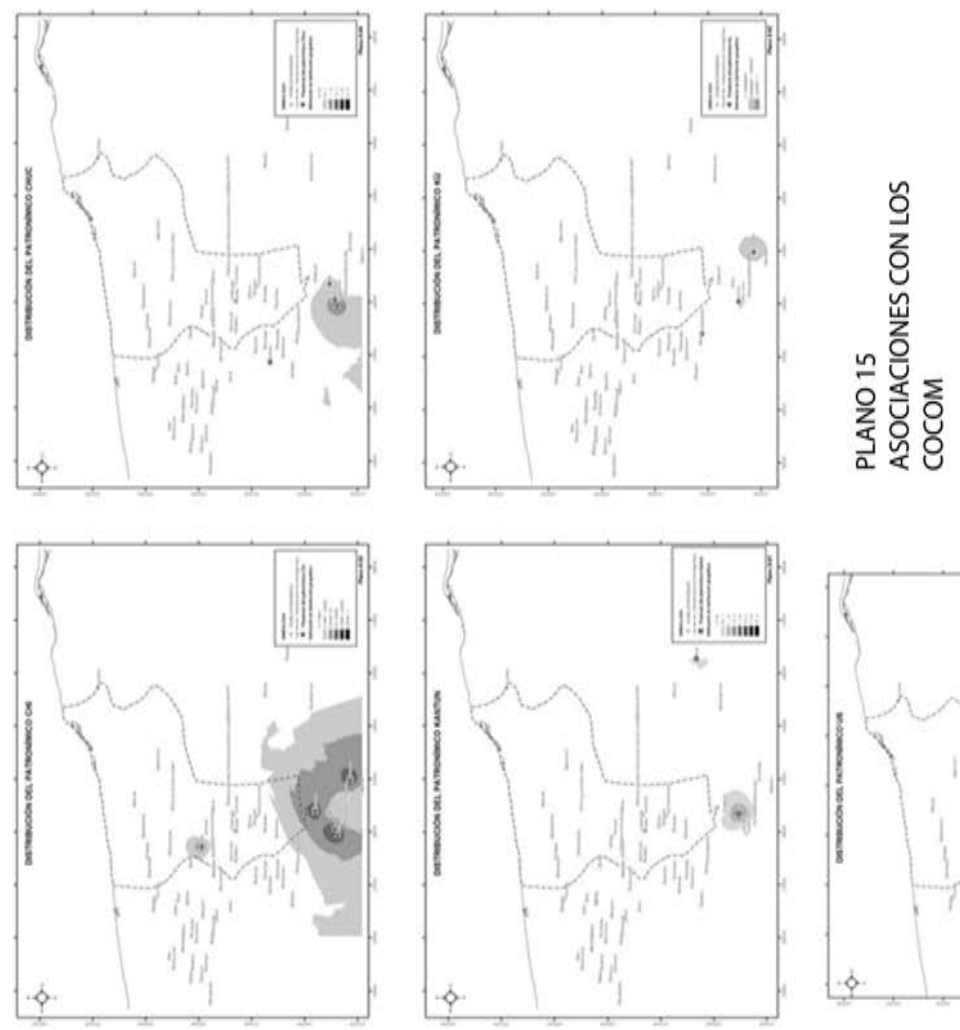

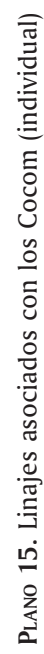
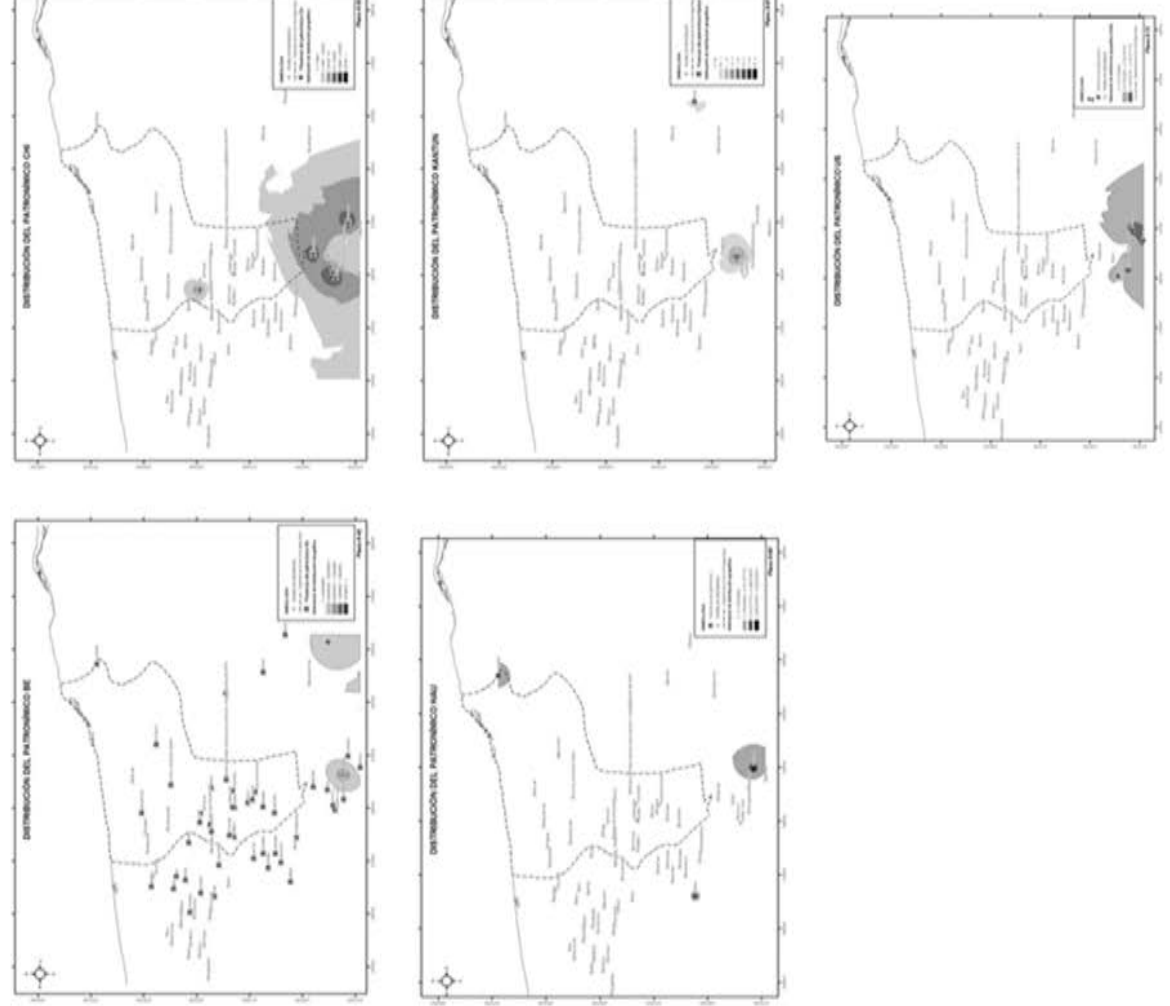
mientras que en el caso de los Cupul, a pesar de que no había unidad política, el predominio político e influencia del linaje sobre otros era avasallador.

Por el otro lado, tenemos linajes de probable origen foráneo, luit y Ch'el, con un patrón focalizado y que establecen estrategias de control basadas en una interacción mayor con la nobleza local al interior de cada pueblo. Este proceso de consolidación del poder a través de alianzas políticas se refleja tanto en la distribución geográfica de los linajes principales como en los relatos históricos. En el caso de los Ch'el, su discurso de origen y legitimación replica los elementos utilizados por los Xiu y los primeros gobernantes de Mayapán para sustentar un poder legítimo acorde a la cosmovisión maya. La utilización de estrategias como los vínculos matrimoniales por vía femenina y el ofrecimiento temporal de "servicios", documentadas en sitios contemporáneos del centro de México, invita a reflexionar sobre los llamados "contactos e influencias" a nivel mesoamericano y la forma como se estaban reconstituyendo los sistemas políticos locales antes de la llegada de los españoles.

Finalmente, el caso de los Cocom establece interesantes interrogantes sobre la línea de investigación aquí seguida. Este linaje mostró tanto elementos locales como foráneos en su estructura de organización, lo que puede ir en apoyo de las versiones históricas que describen una refundación del señorío de los Cocom, después de su expulsión de Mayapán.

Un paso siguiente en esta investigación será la aplicación de este tipo de análisis a nivel peninsular para corroborar las tendencias observadas y comparar el comportamiento de otros linajes relevantes en la historia Posclásica de la península como los Xiu y los Canul.

\section{BIBLIOGRAFÍA}

Barrera Vásquez, Alfredo y Silvia Rendón

1948 El libro de los libros del Chilam Balam. México: Fondo de Cultura Económica.

Brinton, Daniel G.

1969 Maya Chronicles. Library of Aboriginal American Literature, Filadelfia / Nueva [1882] York / AMS Press.

Calepino Maya de Motul

2001 Atribuido a Antonio de Ciudad Real, René Acuña (ed.). México: Plaza y Valdés.

Ciudad Real, Antonio de

1995 Calepino de Motul: Diccionario maya-español, Ramón Arzápalo Marín (ed.). México: Universidad Nacional Autónoma de México, Instituto de Investigaciones Antropológicas, 3 vols. 
Códice Pérez

1949 E. Solís Alcalá (trad.). Mérida [México]: Liga de la Acción Social.

Ah Nakuk Pech. História y crónica de Chac-Xulub-Chen

1936 Héctor Pérez Martínez (ed.). México: Secretaría de Educación Pública.

Crónica de Yaxkukul

1574 Manuscrito maya original en Nueva Orleans: Tulane University, Howard Tilton Memorial Library Latin American Library, 26 (Yucatan Collection Box 1 Folder 3, 4, 5, 6, 7).

"Crónica de Yaxkukul"

1926 En Crónicas Mayas. Juan Martínez Hernández (trad.), Carlos R. Menendez (ed.). Mérida [México]: Compañía Tipográfica Yucateca.

Cruz Cortés, Noemí

2005 Las señoras de la Luna. México: Universidad Nacional Autónoma de México, Instituto de Investigaciones Filológicas, Centro de Estudios Mayas (Cuadernos del Centro de Estudios Mayas, 32).

Demarest, Arthur

1992 "Ideology in Ancient Maya Cultural Evolution: The Dynamics of Galactic Polities", Ideology and Pre-Columbian Civilizations. A. Demarest y G.W. Conrad (eds.). Santa Fe [Estados Unidos]: A School of American Research Press, 135158.

Diccionario de Autoridades

2002 Real Academia Española. Edición facsímil. Madrid: Gredos. 3 vols.

[1732]

Diccionario Maya, maya-español/español-maya

2001 Alfredo Barrera Vásquez (dir.). México: Porrúa.

Durán, fray Diego

$1995 \quad$ Historia de las Indias de Nueva España e Islas de Tierra Firme. México: Consejo Nacional para la Cultura y las Artes, 2 vols.

Grube, Nikolai

1990 Hieroglyphic Sources for the History of Northwest Yucatán. Ponencia presentada en Bonn First Maler Symposium.

Grube, Nikolai y Simon Martin

1998 "Política clásica maya dentro de una tradición mesoamericana: un modelo epigráfico de organización política hegemónica”, Modelos de entidades políticas mayas, primer seminario de las Mesas Redondas de Palenque, S. Trejo (ed.). México: Consejo Nacional para la Cultura y las Artes / Instituto Nacional de Antropología e Historia, 131-146. 
Kepecs, Susan M.

1999 "The Political Economy of Chikinchel, Yucatan, Mexico: A Diachronic Analysis from the Pre-Hispanic Era through the Age of Spanish Administration”, tesis de doctorado en Antropología. Madison: University of Wisconsin, Department of Anthropology.

Kettunen Harri y Christopher Helmke

2010 La escritura jeroglífica maya. Acta Ibero-Americana Fennica. Madrid: Instituto Iberoamericano de Finlandia (Series Hispano-Americano 8).

Landa, fray Diego de

1938 Relación de Las Cosas de Yucatán sacada de lo que escribió el padre Fray Diego de Landa de la orden de San Francisco MDLXVI. Mérida: E.G. Triay e Hijos.

Lizana, Bernardo de

1995 Devocionario de Nuestra Señora de Izamal y conquista espiritual de Yucatán. René

[1633] Acuña (ed.). México: Universidad Nacional Autónoma de México.

Marcus, Joyce

1973 "Territorial Organization of the Lowland Maya", Science 180: 911-916. Vancouver: American Association for the Advancement of Science.

1976 Emblem and State in the Classic Maya Lowlands. An Epigraphic Approach to Territorial Organization. Harvard: Dumbarton Oaks, Trustees for Harvard University.

1993 "Ancient Maya Political Organization", Lowland Maya Civilization in the Eighth Century A.D., J.A. Sabloff y J.S. Henderson (eds.). Washington, DC: Dumbarton Oaks Research Library and Collection, 111-183.

Martin, Simon y Nikolai Grube

1995 "Maya Superstates", Archaeology, 48 (6): 41-46. New York: Archaeological Institute of America.

2000 Chronicle of the Maya Kings and Queens. Deciphering the Dinasties of the Ancient Maya. Nueva York: Thames and Hudson.

Molina, fray Alonso de

1992 Vocabulario en lengua castellana y mexicana y mexicana y castellana. México:

[1571] Porrúa.

Molina Solís, Juan Francisco

1943 Historia del descubrimiento y conquista de Yucatán con una reseña de la historia

[1896] de los mayas. México: Ediciones Mensaje, 2 vols.

Okoshi Harada, Tsubasa

1992 "Los Canules: análisis etnohistórico del Códice de Calkiní”, tesis de doctorado en Historia. México: Universidad Nacional Autónoma de México, Facultad de Filosofía y Letras.

1994 "Ecab: una revisión de la geografía política de una provincia maya yucateca”, Memoria del Primer Congreso Internacional de Mayistas, V. III, México: 
Universidad Nacional Autónoma de México, Instituto de Investigaciones Filológicas, Centro de Estudios Mayas, 280-287.

1996 Volviendo del inframundo: una reconsideración del episodio de Hunac Ceel registrado en el Chilam Balam de Chumayel. Ponencia presentada en la XXIV Mesa Redonda de la Sociedad Mexicana de Antropología. Tepic, del 4 al 11 de agosto de 1996. Texto facilitado por el autor.

1997 Organización político territorial de los Cupul y los Canul: un estudio de Caso. Ponencia presentada en The 62nd Annual Meeting of Society for American Archaeology, Nashville, Tennessee, 2-6 de abril de 1997.

2000 "Los Xiu del siglo xvi: una lectura de dos textos mayas coloniales", Mesoamérica 39 (junio de 2000): 225-238. Wellfleet [Estados Unidos]: Plumsock Mesoamerican Studies.

Pérez Rivas, Manuel E.

2009 "Patrón de asentamiento y organización político territorial en la provincia prehispánica de Ah Kin Ch’el, Yucatán, México”, tesis de doctorado en Estudios Mesoamericanos. México: Universidad Nacional Autónoma de México, Facultad de Filosofía y Letras.

Quezada, Sergio

1993 Pueblos y Caciques Yucatecos, 1550-1580. México: El Colegio de México.

Relaciones histórico-geográficas de la gobernación de Yucatán

1983 Mercedes de la Garza, Ana Luisa Izquierdo, Carmen León y Tolita Figueroa (eds.), 2 vols. México: Universidad Nacional Autónoma de México, Centro de Estudios Mayas.

Restall, Mathew

2006 "Origin and Myth: Ethnicity, Class, and Chibal in Postclassic and Colonial Yucatan”, Nuevas perspectivas sobre la geografía política de los mayas, T. Okoshi, L. Williams-Beck y A. L. Izquierdo (eds.). México: Universidad Nacional Autónoma de México / Universidad Autónoma de Campeche / Fundación para el Avance de los Estudios Mesoamericanos, 269-289.

Reyes García, Luis

1988 Cuauhtinchán del siglo XII al XVI: formación y desarrollo histórico de un señorío prehispánico. México: Fondo de Cultura Económica.

Roys, Ralph L.

1933 The Book of Chilam Balam of Chumayel. Washington, D.C.: Carnegie Institution of Washington (Publication 438).

1940 "Personal Names of the Maya of Yucatan", Contributions to American Anthropology and History, 31: 32-48. Washington, D.C.: Carnegie Institution of Washington (Publication 523)

1957 The Political Geography of the Yucatan Maya. Washington, D.C.: Carnegie Institution of Washington (Publication 613).

1962 "Literary Sources for the History of Mayapan", Mayapan, Yucatan Mexico: 2586. Washington, D.C.: Carnegie Institution of Washington (Publication 619). 
Sabloff, Jeremy A. y David A. Freidel

1975 "A model of a Pre-Columbian trading center", Ancient Civilization and Trade: 369-408; Jeremy A. Sabloff y C.C. Lamberg-Karlovsky (eds.). Alburquerque: University of New Mexico Press.

Sharer, Robert J.

1994 The Ancient Maya. Stanford [Estados Unidos]: Stanford University Press.

Schele, Linda y David Freidel

1990 A Forest of Kings, The Untold History of the Ancient Maya. Nueva York: Quill William Morrow.

Scholes, F.V. y E.B. Adams

1938 Don Diego Quijada, Alcalde Mayor de Yucatán, 1561-1565. México: Antigua librería Robredo, de J. Porrúa e hijos, 2 vols. (Biblioteca Histórica Mexicana de obras inéditas 14 y 15).

Thompson, J. Eric. S.

1984 Historia y religión de los mayas. México: Siglo XXI (Col. América Nuestra, 7).

Torquemada, Fray Juan de

1975 Monarquía Indiana. México: Universidad Nacional Autónoma de México, Ins-

[1727] tituto de Investigaciones Históricas, 7 vols.

Voss, Alexander y H. Juergen Kremer

2000 'K'ak'-u-pakal, Hun-pik-tok' and the Kokom: The Political Organization of Chichén Itzá", The Sacred and the Profane: Architecture and Identity in the Maya Lowlands, acta Mesoamericana, 10: 149-181, Pierre Robert Colas et al. (eds.). Möckmühl [Alemania]: Verlag Anton Saurwein.

Yates Sosa, Rafael

1994 Diccionario onomástico de Mérida, Yucatán: desde su fundación hasta 1994. Mérida: Gobierno del Estado de Yucatán/Talleres Gráficos del Sudeste.

Documentos consultados

1567 Carta en donde los caciques piden a SM., religiosos de la orden de San Francisco. Referencia AGI, Audiencia de México, Leg. 367. (Referencia ES.41091. AGI/1.16403.13.369//MEXICO,367)

Tasaciones de los pueblos de la provincia de Yucatán hechas por la audiencia de Santiago de Guatemala en el mes de febrero de 1549 (AGI, Papeles de Simancas, Caj. 6, Leg. 1) (AGI, Audiencia de Guatemala, Leg. 128, ff. 307-401v).

Residencia tomada al doctor Diego de Quijada, del tiempo que fue gobernador de la provincia de Yucatán, Cozumel y Tabasco, por Luis de Céspedes y Oviedo, juez nombrado para este efecto y gobernador de dichas provincia ( AGI, Justicia 245, f 1001v-1562v. (Referencia ES.41091.AGI/23.8.26//JUSTICIA,245). 